Draft Version June 29, 2018

Preprint typeset using IATEX style emulateapj v. 08/13/06

\title{
VARIABLE PROTOSTELLAR ACCRETION WITH EPISODIC BURSTS
}

\author{
Eduard I. VOROBYOV ${ }^{1,2}$ AND Shantanu Basu ${ }^{3}$ \\ Draft version June 29, 2018
}

\begin{abstract}
We present the latest development of the disk gravitational instability and fragmentation model, originally introduced by us to explain episodic accretion bursts in the early stages of star formation. Using our numerical hydrodynamics model with improved disk thermal balance and star-disk interaction, we computed the evolution of protostellar disks formed from the gravitational collapse of prestellar cores. In agreement with our previous studies, we find that cores of higher initial mass and angular momentum produce disks that are more favourable to gravitational instability and fragmentation, while a higher background irradiation and magnetic fields moderate the disk tendency to fragment. The protostellar accretion in our models is time-variable, thanks to the nonlinear interaction between different spiral modes in the gravitationally unstable disk, and can undergo episodic bursts when fragments migrate onto the star owing to the gravitational interaction with other fragments or spiral arms. Most bursts occur in the partly embedded Class I phase, with a smaller fraction taking place in the deeply embedded Class 0 phase and a few possible bursts in the optically visible Class II phase. The average burst duration and mean luminosity are found to be in good agreement with those inferred from observations of FU-Orionis-type eruptions. The model predicts the existence of two types of bursts: the isolated ones, showing well-defined luminosity peaks separated with prolonged periods $\left(\sim 10^{4} \mathrm{yr}\right)$ of quiescent accretion, and clustered ones, demonstrating several bursts occurring one after another during just a few hundred years. Finally, we estimate that $40 \%-70 \%$ of the star-forming cores can display bursts after forming a star-disk system.
\end{abstract}

Subject headings: accretion, accretion disks - hydrodynamics - instabilities - ISM: clouds - stars: formation

\section{INTRODUCTION}

Low-mass stars form as a result of the gravitational collapse of dense gaseous cores. Standard models of core collapse predict that the accretion rate onto a forming protostar is proportional to the cube of the sound speed (Larson 1969; Penston 1969; Shu 1977). When a finite size of the core is taken into account, numerical simulations of gravitationally unstable, spherically symmetric cores indicate that accretion is tapering off with time in the late evolution (Foster \& Chevalier 1993; Vorobyov \& Basu 2005). However, when even a modest degree of rotation is present initially in the core as suggested by observations (e.g. Caselli et al. 2002), simple arguments based on the centrifugal radius and sophisticated numerical hydrodynamics simulations both demonstrate that most of the core mass does not fall directly onto the protostar but rather lands onto an accretion disk formed from conservation of angular momentum of the core.

It has recently become evident that the mass infall rate onto the disk $\dot{M}_{\text {infall }}$ at radial scales on the order of $1000 \mathrm{AU}$ and the mass accretion rate onto the star $\dot{M}$ may be significantly different thanks to the complicated interplay of various physical mechanisms of mass and angular momentum transport operating in the disk. For instance, $\dot{M}_{\text {infall }}$ in isolated core models gradually

\footnotetext{
${ }^{1}$ Department of Astrophysics, The University of Vienna, Vienna, 1180, Austria; eduard.vorobiev@univie.ac.at.

${ }^{2}$ Research Institute of Physics, Southern Federal University, Stachki 194, Rostov-on-Don, 344090, Russia.

${ }^{3}$ Department of Physics and Astronomy, University of Western Ontario, London, Ontario, N6A 3K7, Canada; basu@uwo.ca.
}

declines with time from a few $\times 10^{-6} M_{\odot} \mathrm{yr}^{-1}$ to a negligible value by the end of the embedded phase, but $\dot{M}$ in gravitationally unstable disks can be highly variable (Vorobyov 2009; Rice et al. 2010), sometimes exhibiting episodic accretion bursts $\gtrsim 10^{-4} M_{\odot} \mathrm{yr}^{-1}$ caused by disk gravitational fragmentation and migration of the fragments onto the protostar (Vorobyov \& Basu 2005, 2006, 2010a). The formation of giant planets in the disk can also significantly alter the character of accretion, creating various patterns of variability and bursts exceeding in magnitude $10^{-3} M_{\odot} \mathrm{yr}^{-1}$ (Machida et al. 2011; Nayakshin \& Lodato 2012). A combination of the magneto-rotational and thermal instabilities in the inner several AU and gravitational instability further out in the disk (or layered accretion) was shown to produce accretion bursts typical for FU-Orionis-type eruptions (Armitage et al. 2001; Zhu et al. 2009). To complicate the things further, $\dot{M}_{\text {infall }}$ may experience significant variations when the chaotic and turbulent nature of clustered star formation is taken into consideration (e.g. Bate et al. 2010; Padoan et al. 2014).

Observations support the growing evidence that accretion onto low-mass protostars is at least partly variable. An ever growing number of FU-Orionis-type and EXLupi-like eruptive stars (Audard et al. 2014) does not fit into the standard models of spherical core collapse. The mean/median luminosity of protostars in young starforming regions appears to be lower by about an order of magnitude than that predicted from the standard models (e.g. Kenvon et al. 1990; Evans et al. 2009). Accretion rates gradually declining in time and showing episodic bursts were shown to resolve this "luminosity problem" 
(Dunham \& Vorobyov 2012). Monitoring of the accretion variability suggest that about half of all protostars show up to $50 \%$ variations in $\dot{M}$ over timescales less than 2 yr (Billot et al. 2012). Surveys of young stars in starforming regions and in the Galactic plane revealed that about $0.1 \%$ of objects show a luminosity increase by more than a factor of 2.5 over 5 yr (Scholz et al.|2013), including recent flares in VSX J205126.1 (Kóspál et al. 2011) and V1647 Ori (Ábrahám et al. 2004). The knotted morphology of jets seen in some protostellar systems suggests an underlying variability in the mass accretion, although the combination of jet velocities and spacing between the knots often suggest shorter periods of episodicity than predicted for FU-Orionis-type stars (Arce et al. 2013).

To summarize, accretion onto young stars seems to exhibit a variety of patterns with time variations of different amplitude and duration and, as noted in Dunham \& Vorobyov (2012), is better termed as variable accretion with episodic bursts. This newly emerging paradigm is beginning to supersede the classical ShuLarson-Penston steady accretion models and may have important consequences for the evolution of stars and planets. For instance, variable accretion can help to explain the luminosity spread of young clusters without invoking a significant age spread (Baraffe et al. 2009, 2012). In addition, quiescent periods between accretion bursts can promote disk fragmentation and giant planet formation (Stamatellos 2011). Finally, variable accretion with episodic bursts is expected to have a significant impact on the disk and envelope chemistry and on the composition of ices in protostellar disks (Lee 2007; Visser \& Bergin 2012; Kim et al. 2012)

In this paper, we revisit the disk instability and fragmentation model for episodic accretion and luminosity bursts, originally developed by us in a series of papers (Vorobyov \& Basu 2005, 2006, 2010a), using an improved numerical hydrodynamics code which takes into account a better disk thermal physics, improved dust opacities, and an accurate calculation of the stellar photospheric and accretion luminosities using a stellar evolution code that takes stellar accretion into account. The latter update allows us to calculate the burst statistics and perform direct comparison with observations, and also make prediction regarding the expected fraction of starforming cores that can display bursts after forming a star-disk system. The paper is organized as follows. A brief description of the numerical model and recent updates are presented in Section 2. The main results are described in Section 3. The characteristics of the bursts obtained in the framework of our model are reviewed in Section 4. The time evolution of individual bursts is considered in Section 6. The expected fraction of starforming cores than can exhibit bursts after forming a star-disk systems is calculated in Section 7 and main conclusions are summarized in Section 8

\section{MODEL DESCRIPTION}

Our numerical model is described in detail in Vorobyov \& Basu (2010a) and is briefly reviewed below with the emphasis on several recent updates. We start our numerical simulations from the gravitational collapse of a starless cloud core, continue into the embedded phase of star formation, during which a star, disk, and envelope are formed, and terminate our simulations when the age of the star becomes older than 1.0 Myr. Such long integration times are made possible by the use of the thindisk approximation, the justification of which is provided in Vorobyov \& Basu (2010a). The protostellar disk occupies the inner part of the numerical polar grid and is exposed to intense mass loading from the infalling envelope.

To avoid too small time steps, we introduce a "sink cell" at $r_{\mathrm{sc}}=6.0 \mathrm{AU}$ and impose a free inflow inner boundary condition and a free outflow outer boundary condition so that the matter is allowed to flow out of the computational domain but is prevented from flowing in. The sink cell is dynamically inactive; it contributes only to the total gravitational potential and secures a smooth behaviour of the gravity force down to the stellar surface. During the early stages of the core collapse, we monitor the gas surface density in the sink cell and when its value exceeds a critical value for the transition from isothermal to adiabatic evolution, we introduce a central point-mass object. In the subsequent evolution, $90 \%$ of the gas that crosses the inner boundary is assumed to land on the central object. The other $10 \%$ of the accreted gas is assumed to be carried away with protostellar jets.

\subsection{Main equations}

The basic equations of mass, momentum, and energy transport in the thin-disk limit are

$$
\begin{gathered}
\frac{\partial \Sigma}{\partial t}=-\nabla_{p} \cdot\left(\Sigma \boldsymbol{v}_{p}\right), \\
\frac{\partial}{\partial t}\left(\Sigma \boldsymbol{v}_{p}\right)+\left[\nabla \cdot\left(\Sigma \boldsymbol{v}_{\boldsymbol{p}} \otimes \boldsymbol{v}_{p}\right)\right]_{p}=-\nabla_{p} \mathcal{P}+\Sigma \boldsymbol{g}_{p}+(2) \\
+(\nabla \cdot \boldsymbol{\Pi})_{p},-\nabla_{p}\left(\frac{B_{z}^{2}}{4 \pi} Z\right)+\frac{B_{z} \boldsymbol{B}_{p}^{+}}{2 \pi} \\
\frac{\partial e}{\partial t}+\nabla_{p} \cdot\left(e \boldsymbol{v}_{p}\right)=-\mathcal{P}\left(\nabla_{p} \cdot \boldsymbol{v}_{p}\right)-\Lambda+\Gamma+(\nabla \boldsymbol{v})_{p p^{\prime}}: \Pi_{p p^{\prime}},
\end{gathered}
$$

where subscripts $p$ and $p^{\prime}$ refers to the planar components $(r, \phi)$ in polar coordinates, $\Sigma$ is the mass surface density, $e$ is the internal energy per surface area, $\mathcal{P}$ is the vertically integrated gas pressure calculated via the ideal equation of state as $\mathcal{P}=(\gamma-1) e, Z$ is the radially and azimuthally varying vertical scale height determined in each computational cell using an assumption of local hydrostatic equilibrium, $\boldsymbol{v}_{p}=v_{r} \hat{\boldsymbol{r}}+v_{\phi} \hat{\boldsymbol{\phi}}$ is the velocity in the disk plane, and $\nabla_{p}=\hat{\boldsymbol{r}} \partial / \partial r+\hat{\boldsymbol{\phi}} r^{-1} \partial / \partial \phi$ is the gradient along the planar coordinates of the disk. The gravitational acceleration in the disk plane, $\boldsymbol{g}_{p}=$ $g_{r} \hat{\boldsymbol{r}}+g_{\phi} \hat{\boldsymbol{\phi}}$, takes into account self-gravity of the disk, found by solving for the Poisson integral (see details in Vorobyov \& Basu 2010a), and the gravity of the central protostar when formed. Turbulent viscosity is taken into account via the viscous stress tensor $\boldsymbol{\Pi}$, the expression for which is provided in Vorobyov \& Basu (2010a). We parameterize the magnitude of kinematic viscosity $\nu$ using the $\alpha$-prescription with a spatially and temporally uniform $\alpha$.

Two 1.h.s. terms in Equation (2) represent magnetic pressure and tension in the thin-disk approximation, where $B_{z}$ is the vertically uniform magnetic field in the disk and $\boldsymbol{B}_{p}^{+}=B_{r}^{+} \hat{\boldsymbol{r}}+B_{\phi}^{+} \hat{\phi}$ are the planar 
components of the magnetic field at the top surface of the disk. In the flux-freezing approximation adopted in this work the vertical magnetic field component in the disk can be determined from the relation $B_{z}=$ $2 \pi G^{1 / 2} \Sigma / \mu_{\mathrm{B}}$ (Nakano \& Nakamura 1978), where $\mu_{\mathrm{B}}$ is the spatially uniform mass-to-flux ratio. The planar components of the magnetic field are directly related to the planar components of gravitational acceleration $\boldsymbol{g}_{p}$ through the following relation $B_{z} \boldsymbol{B}_{p}^{+} /(2 \pi)=-\boldsymbol{g}_{p} / \mu_{\mathrm{B}}^{2}$ (see Vorobyov \& Basu 2006, for more details).

The radiative cooling $\Lambda$ in equation (3) is determined using the diffusion approximation of the vertical radiation transport in a one-zone model of the vertical disk structure (Johnson \& Gammie 2003)

$$
\Lambda=\mathcal{F}_{\mathrm{c}} \sigma T_{\mathrm{mp}}^{4} \frac{\tau}{1+\tau^{2}},
$$

where $\tau$ is the optical depth to the disk midplane, $\sigma$ is the Stefan-Boltzmann constant, $T_{\mathrm{mp}}=\mathcal{P} \mu / R \Sigma$ is the midplane temperature of gas $^{4}, \mu=2.33$ is the mean molecular weight, $R$ is the universal gas constant, and $\mathcal{F}_{\mathrm{c}}=2+20 \tan ^{-1}(\tau) /(3 \pi)$ is a function that secures a correct transition between the optically thick and optically thin regimes. The heating function is expressed as

$$
\Gamma=\mathcal{F}_{\mathrm{c}} \sigma T_{\mathrm{irr}}^{4} \frac{\tau}{1+\tau^{2}},
$$

where $T_{\text {irr }}$ is the irradiation temperature at the disk surface determined by the stellar and background blackbody irradiation as

$$
T_{\mathrm{irr}}^{4}=T_{\mathrm{bg}}^{4}+\frac{F_{\mathrm{irr}}(r)}{\sigma},
$$

where $T_{\mathrm{bg}}$ is the uniform background temperature (in our model set to the initial temperature of the natal cloud core) and $F_{\text {irr }}(r)$ is the radiation flux (energy per unit time per unit surface area) absorbed by the disk surface at radial distance $r$ from the central star. The latter quantity is calculated as

$$
F_{\text {irr }}(r)=\frac{L_{*}}{4 \pi r^{2}} \cos \gamma_{\text {irr }},
$$

where $\gamma_{\text {irr }}$ is the incidence angle of radiation arriving at the disk surface (with respect to the normal) at radial distance $r$. The stellar luminosity $L_{*}$ is the sum of the accretion luminosity $L_{*, \text { accr }}=(1-\epsilon) G M_{*} \dot{M} / 2 R_{*}$ arising from the gravitational energy of accreted gas and the photospheric luminosity $L_{*, \text { ph }}$ due to gravitational compression and deuterium burning in the stellar interior. The stellar mass $M_{*}$ and accretion rate onto the star $\dot{M}$ are determined using the amount of gas passing through the sink cell, while the stellar radius $R_{*}$ is returned by a stellar evolution code (see Section 2.2 for details and the definition of $\epsilon$ ). Equations (10-(3) are solved in the polar coordinates on a numerical grid with $512 \times 512$ grid zones. The solution procedure is described in detail in Vorobyov \& Basu (2010a).

\subsection{Recent updates}

\footnotetext{
4 This definition of the midplane temperature is accurate within a factor of unity (Zhu et al. 2012)
}

In this study, several important updates have been implemented to the numerical code as compared to the earlier work of Vorobyov \& Basu (2010a). First, we have implemented newer Semenov dust opacities (Semenov et al. 2003) instead of older Bell \& Lin opacities (Bell \& Lin 1994). The Semenov opacities are somewhat higher than those of Bell \& Lin in the temperature range typical for protostellar disks, which results in a somewhat higher gas temperature. Second, we considered a stiffer equation of state taking into account the fact that the rotational and vibrational degrees of freedom of molecular hydrogen are excited only above $100 \mathrm{~K}$ (e.g. Masunaga \& Inutsuka 2000). As a result, the ratio of specific heats takes the following from

$$
\gamma= \begin{cases}5 / 3, & \text { if } T_{\mathrm{g}}<100 \mathrm{~K} \\ 7 / 5, & \text { if } 100 \mathrm{~K} \leq T_{\mathrm{g}}<2000 \mathrm{~K}, \\ 1.1, & \text { if } T_{\mathrm{g}} \geq 2000 \mathrm{~K} .\end{cases}
$$

In Vorobyov \& Basu (2010a), $\gamma$ was set to $7 / 5$ for $T_{\mathrm{g}}<$ $2000 \mathrm{~K}$. The net result is an overall moderate increase in the disk temperature at distances $\gtrsim 10 \mathrm{AU}$.

The aforementioned updates enable a better calculation of the thermal balance in the disk and hence a more accurate study of the gravitational instability and fragmentation. We note that the net effect of these updates is an increase in the disk temperature, making disk fragmentation more difficult.

Finally, we have improved on the method in which the parameters of the central star, are calculated. In Vorobyov \& Basu (2010a), pre-main-sequence tracks for the non-accreting low-mass stars and brown dwarfs of D'Antona \& Mazzitelli (1994) were employed to calculate the stellar photospheric luminosity and radius. In this work, the properties of the forming protostar are calculated using a stellar evolution code described in Baraffe \& Chabrier (2010). As in Baraffe et al. (2012), we assume that the fraction $\epsilon$ of the accretion energy $G M_{*} \dot{M} /\left(2 R_{*}\right)$ is absorbed by the protostar, while the fraction $(1-\epsilon)$ is radiated away and contributes to the accretion luminosity of the star $L_{* \text {,accr. Despite many }}$ efforts, the exact value of $\epsilon$ in low-mass star formation is not known. In the present calculations, we adopt a socalled "hybrid" scheme (see Baraffe et al. 2012, for detail) with $\epsilon=0$ when accretion rates remain smaller than a critical value $\dot{M}_{\mathrm{cr}}=10^{-5} M_{\odot} \mathrm{yr}^{-1}$, and $\epsilon=0.2$ when $\dot{M}>\dot{M}_{\text {cr }}$.

The stellar evolution code is coupled with the main hydrodynamical code in real time. The input parameter to the stellar evolution code provided by disk modeling is the mass accretion rate onto the star $\dot{M}$. The output of the stellar evolution code are the stellar radius $R_{*}$ and the photospheric luminosity $L_{*, \mathrm{ph}}$, which are employed by the disk hydrodynamics simulations to calculate the total stellar luminosity and the radiation flux reaching the disk surface. Due to heavy computational load the stellar evolution code is invoked to update the properties of the protostar only every $20 \mathrm{yr}$, while the hydrodynamical time step may be as small as a few weeks and the entire duration of numerical simulations may exceed 1.0 Myr.

The coupling of disk modeling with the stellar evolution code is essential. As was demonstrated in Baraffe et al. (2009, 2012), the stellar properties derived 
TABLE 1

MODEL PARAMETERS

\begin{tabular}{cccccccccc}
\hline \hline Model & $\begin{array}{c}M_{\text {core }} \\
\left(M_{\odot}\right)\end{array}$ & $\begin{array}{c}\beta \\
(\%)\end{array}$ & $\begin{array}{c}T_{\text {init }} \\
(\mathrm{K})\end{array}$ & $\begin{array}{c}\Omega_{0} \\
\left(\mathrm{~km} \mathrm{~s}^{-1} \mathrm{pc}^{-1}\right)\end{array}$ & $\begin{array}{c}r_{0} \\
(\mathrm{AU})\end{array}$ & $\begin{array}{c}\Sigma_{0} \\
\left(\mathrm{~g} \mathrm{~cm}^{-2}\right)\end{array}$ & $\begin{array}{c}R_{\text {out }} \\
(\mathrm{pc})\end{array}$ & $\alpha$ & $\mu_{\mathrm{B}}$ \\
\hline 1 & 1.1 & 0.88 & 10 & 1.4 & 2400 & $5.2 \times 10^{-2}$ & 0.07 & $5 \times 10^{-3}$ & 0 \\
2 & 1.5 & 0.88 & 10 & 1.0 & 3400 & $3.7 \times 10^{-2}$ & 0.1 & $5 \times 10^{-3}$ & 0 \\
3 & 0.31 & 0.88 & 10 & 3.3 & 685 & $1.8 \times 10^{-1}$ & 0.03 & $5 \times 10^{-3}$ & 0 \\
4 & 1.1 & 0.14 & 10 & 0.57 & 2400 & $5.2 \times 10^{-2}$ & 0.07 & $5 \times 10^{-3}$ & 0 \\
5 & 1.1 & 0.88 & 25 & 5.7 & 960 & $3.2 \times 10^{-1}$ & 0.028 & $5 \times 10^{-3}$ & 0 \\
6 & 1.1 & 0.88 & 10 & 1.4 & 2400 & $5.2 \times 10^{-2}$ & 0.07 & $3 \times 10^{-2}$ & 0 \\
7 & 1.1 & 0.88 & 10 & 1.4 & 2400 & $5.2 \times 10^{-2}$ & 0.07 & $5 \times 10^{-3}$ & 3.33 \\
\hline
\end{tabular}

from evolution models that do not take stellar accretion into account (such as D'Antona \& Mazzitelli (1994) data used in our previous work) can significantly differ from those derived from accreting models. For instance, the stellar photospheric luminosities may differ by a factor of up to 10 (see fig. 5 in Baraffe et al. 2012) and stellar radii $^{5}$ by a factor of several (see fig. 2 in Baraffe et al. 2009), depending on the stellar mass and the fraction of accretion energy absorbed by the star $\epsilon$. Therefore, employing accreting stellar evolution models is crucial for an accurate comparison of burst characteristics derived from numerical modeling with those measured in young starforming regions (Sections 4,7). In addition, they can also enable a better calculation of the disk thermal physics, because the main source of heating for flared disks at distances where fragmentation takes place is mostly stellar irradiation (viscosity is more important in the inner several tens of $\mathrm{AU}$ ).

More details on the coupling of the two numerical codes (the hydrodynamic and stellar evolution ones) can be found in Vorobyov et al. (2013).

\subsection{Initial conditions}

For the initial distribution of the gas surface density $\Sigma$ and angular velocity $\Omega$, we adopted those derived by Basu (1997) for pre-stellar cores formed as a result of ambipolar diffusion, with the angular momentum remaining constant during axially-symmetric core compression

$$
\begin{gathered}
\Sigma=\frac{r_{0} \Sigma_{0}}{\sqrt{r^{2}+r_{0}^{2}}}, \\
\Omega=2 \Omega_{0}\left(\frac{r_{0}}{r}\right)^{2}\left[\sqrt{1+\left(\frac{r}{r_{0}}\right)^{2}}-1\right] .
\end{gathered}
$$

Here, $\Omega_{0}$ and $\Sigma_{0}$ are the angular velocity and gas surface density at the center of the core and $r_{0}=\sqrt{A} c_{\mathrm{s}}^{2} / \pi G \Sigma_{0}$ is the radius of the central plateau, where $c_{\mathrm{s}}$ is the initial sound speed in the core. The gas surface density distribution described by equation (9) can be obtained (to within a factor of unity) by integrating the three-dimensional gas density distribution characteristic of Bonnor-Ebert spheres with a positive density-perturbation amplitude A (Dapp \& Basu 2009). In all models the value of the initial density enhancement $A$ is set to 1.2 and all cores have a fixed ratio $r_{\text {out }} / r_{0}=6.0$, where $r_{\text {out }}$ is the radius of the core, implying that the cores are initially unstable to gravitational collapse.

${ }^{5}$ Stellar radius affects the proper calculation of the accretion luminosity.
Individual models are generated by first choosing $r_{\text {out }}$ and then calculating $\Sigma_{0}$. The central angular velocity $\Omega_{0}$ is chosen so as to generate cores with different ratios of rotational to gravitational energy $\beta$, consistent with the values inferred for pre-stellar cores by Caselli et al. (2002). We have considered 7 models, the parameters of which are listed in Table 1. The prototype model 1 has the core mass $M_{\text {core }}$ set to $1.1 M_{\odot}$, the value of $\beta$ set to $0.88 \%$, and the viscous $\alpha$-parameter set to $5 \times 10^{-3}$. The initial gas temperature is $T_{\text {init }}=10 \mathrm{~K}$ and magnetic fields are turned off. Other models are compared against model 1, with their parameters being varied so as to emphasize the effect of different initial core masses, temperatures, and rotation rates, as well as the effect of turbulent viscosity and magnetic field.

\section{VARIABLE ACCRETION WITH EPISODIC BURSTS}

We start with comparing the long-term evolution of circumstellar disks in our non-magnetic models, continue with analysing the accretion rates and the burst statistics in different models, and finish with considering the effect of magnetic fields.

\subsection{Disk evolution}

Figure 1 presents the time evolution of the gas surface density $\Sigma$ in seven models, each row of images corresponding to a particular model. Individual images show the inner region of $2000 \mathrm{AU}$ on each side, whereas the total computational domain is usually much greater. The minimum value of the density scale is set to -1.5 (in log units of $\mathrm{g} \mathrm{cm}^{-2}$ ), a typical value at the disk outer edge (Vorobyov 2010) Four columns show the disk evolution at four representative times $t$ elapsed since the formation of the central protostar.

We start by analyzing the prototype model 1. Evidently, the early disk evolution $(t=0.15-0.25 \mathrm{Myr})$ in this model is characterized by vigourous gravitational instability and fragmentation. The disk has an irregular spiral structure with multiple fragments forming in the densest parts of the arms. As time progresses to $t=0.5-0.75$ Myr, the disk becomes less irregular, taking a smoother shape and exhibiting only a week spiral structure. No fragments are visible in the disk at that time, which does not necessarily mean that disk fragmentation has ceased completely. As will be shown below, this is merely an artifact of infrequent time sampling in Figure 1 and short-lived fragmentation episodes continue to occur even in the late disk evolution.

Nevertheless, the presence of fragments in the early disk evolution and the lack of them in the late evolution implies the existence of efficient mechanisms leading to their loss and/or destruction. Among such 


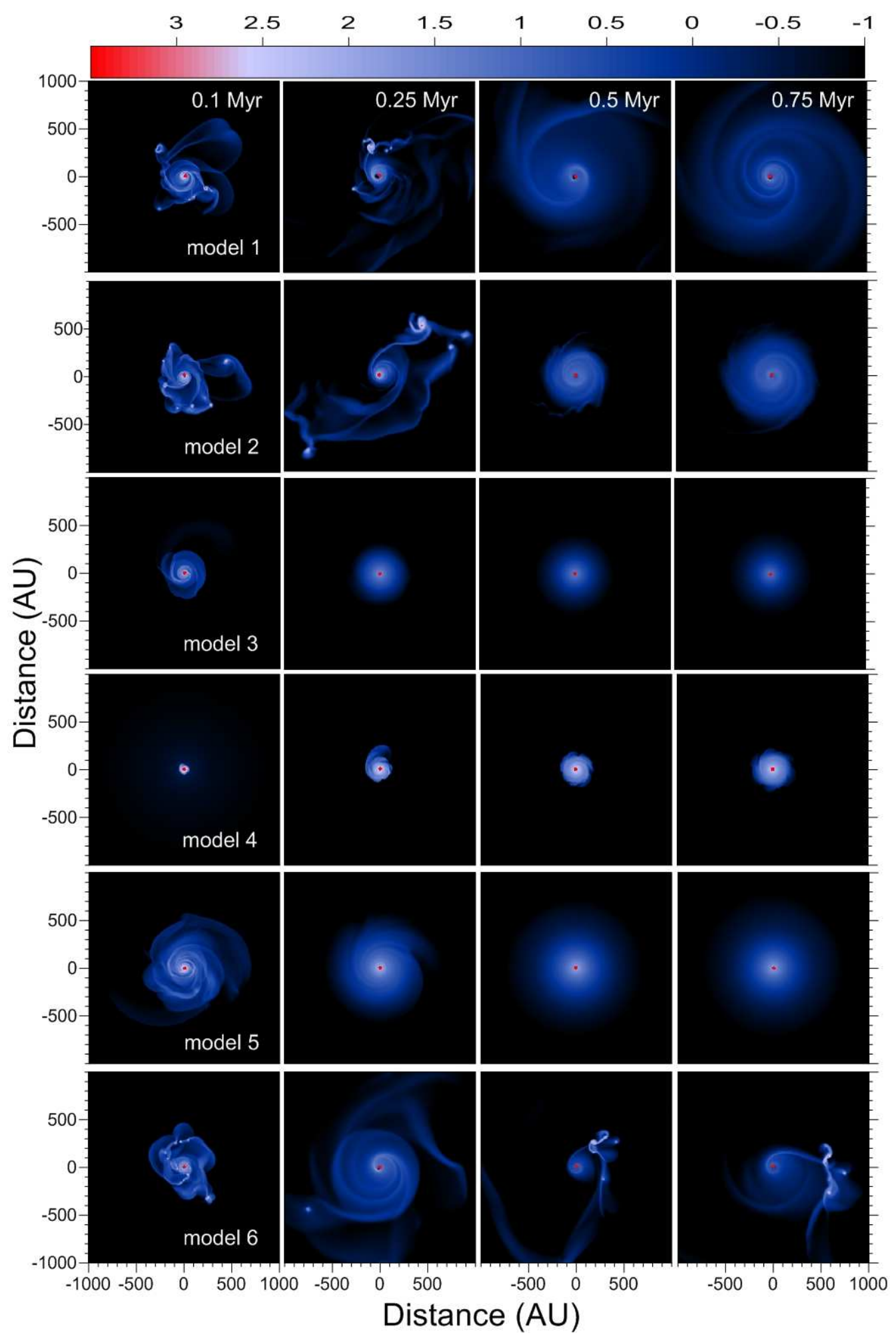

FIG. 1.- Images of the gas surface density in the inner $2000 \times 2000$ AU box in models 1-7 (from top to bottom). Each row represents an individual model at four characteristic times after the formation of the central star. The star is marked by the red circle in the coordinate center. The scale bar is in $\log \mathrm{g} \mathrm{cm}^{-2}$ 
mechanisms are inward migration of fragments onto the protostar caused by the gravitational exchange of angular momentum with spiral arms and other fragments (Vorobvov \& Basu 2006, 2010a; Machida et al. 2011; Tsukamoto et al. 2014), dispersal of fragments due to tidal torques (Bolev et al. 2010; Zhu et al. 2012), and ejection of fragments from the disk into the intracluster medium due to multi-fragment gravitational interaction (Basu \& Vorobyov 2012). In our case, all three mechanisms were found to be at work. In particular, the inward migration and infall of fragments onto the star triggers mass accretion bursts discussed in more detail in Section 3.2 while the ejection of a fragment with mass on the order of $0.1 M_{\odot}$ at $t \approx 0.25 \mathrm{Myr}$ results in a notable drop in the net disk mass and a consequent decrease in the disk fragmentation activity.

The $M_{\text {core }}=1.5 M_{\odot}$ model 2 is characterized by a greater initial core mass than that of model 1 . Nevertheless, the evolution in both models is qualitatively similar - the disk is vigorously unstable to fragmentation in the early evolution showing multiple fragments interconnected with dense spiral filaments, but becomes considerably smoother after $t=0.5 \mathrm{Myr}$. This transformation is caused by the same effect in both models - a fragment is ejected form the disk leading to an appreciable drop in the disk mass and subsequent disk stabilization. The disk size and mass in model 2 at later times are somewhat smaller than those in model 1, but this is a mere consequence of a somewhat more massive fragment being ejected from the disk in model $2\left(\sim 0.25 M_{\odot}\right)$.

On the other hand, model 3 with a smaller initial core mass $M_{\text {core }}=0.3 M_{\odot}$ demonstrates a drastically different evolutionary pattern from that of models 1 and 2 - the disk exhibits a flocculent spiral structure in the early evolution ( $t \leq 0.1 \mathrm{Myr})$ and becomes increasingly axisymmetric with time. No fragmentation is evident in the disk (but see Figure 3 below). Model 3 owes its special behaviour to the fact that cores with lower mass (but with similar $\beta$ ) form lower mass disks. In the case of model 3 , the maximum disk mass is $0.09 M_{\odot}$ at $t \approx 0.1 \mathrm{Myr}$ and it gradually drops to $0.04 M_{\odot}$ at $t=1.0$ Myr. According to figure 1 in Vorobyov (2013), a model with $M_{\text {core }}=0.31 M_{\odot}, \beta=0.88 \%$, and the disk mass of $0.09 M_{\odot}$ lies very near to the fragmentation boundary in the $\beta-M_{\text {core }}$ phase space. This means that model 3 can at best produce a couple of isolated fragmentation episodes, which might have been missed in Figure 1 due to infrequent time sampling.

Model 4 is designed to highlight the disk evolution resulting from the collapse of a cloud core with low angular momentum. In this model, the ratio of rotational to gravitational energy $\beta$ is set to $0.14 \%$, more than six times smaller than that in model 1 , but both models have the same value of the initial core mass, $M_{\text {core }}=1.1 M_{\odot}$. As a direct consequence of the low initial angular momentum in the core, the disk radius in model 4 is rather small $(\approx 50 \mathrm{AU}$ at $t \leq 0.1 \mathrm{Myr}$ and $\lesssim 150 \mathrm{AU}$ in the later evolution), much smaller than in other models. No wonder that there was only one fragment formed at $t=0.36 \mathrm{Myr}$ and that one dispersed after a few orbital period (without reaching the sink cell), possibly due to tidal torques from spiral arms.

Model 5 is set to imitate the effect of a warmer star formation environment with a background radiation tem-

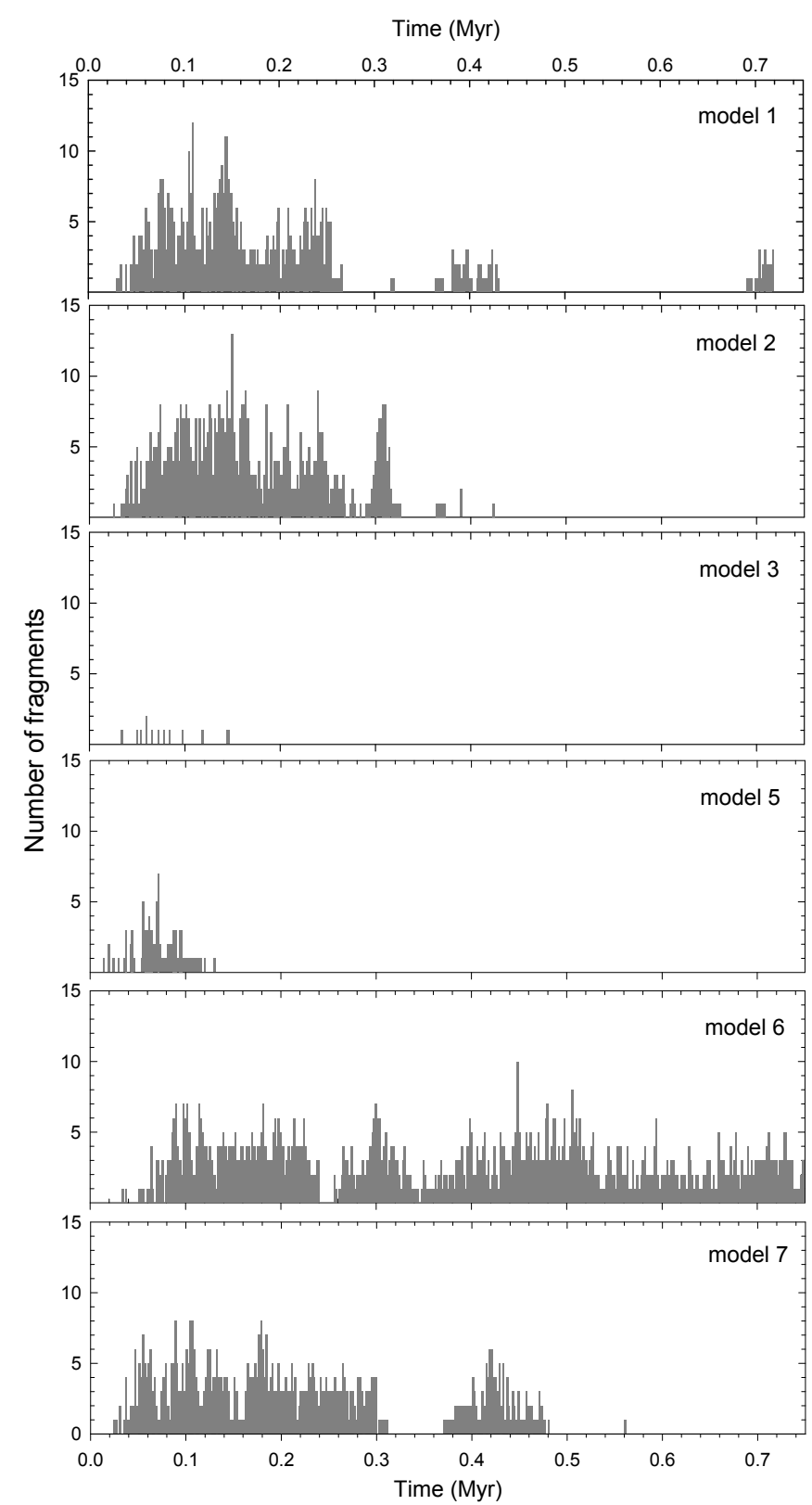

FIG. 2.- Number of fragments vs. time in models 1, 2, 3, 5, 6, and 7 (from top to bottom). The number of fragments at a given time instant is calculated using the fragment tracking algorithm described in Vorobyov et al. (2013). An increase in the number of fragments shows recent fragmentation, and a decrease shows recent destruction/accretion of the fragments. The time is counted since the formation of the protostar.

perature of $T_{\mathrm{bg}}=25 \mathrm{~K}, 2.5$ times higher than that in model 1 . An increase in $T_{\mathrm{bg}}$ has notably stabilized the disk against fragmentation. There are still occasional fragmentation episodes taking place in model 5 (see Figure 2 below), but the disk quickly stabilizes even against gravitational instability (put aside fragmentation!) and becomes virtually axisymmetric after $t=0.5 \mathrm{Myr}$.

The last but one row in Figure 1 presents the disk evolution in model 6 characterized by the $\alpha$-parameter equal to 0.03 , six times greater than in model 1 . The other parameters are identical in both model 6 and 1. A higher efficiency of viscous mass and angular momentum transport does not suppress fragmentation, even though the disk mass decreases from a maximum value of $0.25 M_{\odot}$ in 
model 1 to $0.15 M_{\odot}$ in model 6 . Most curiously, model 6 demonstrates a survival of several fragments orbiting the host star at wide separation orbits ${ }^{6}$. This is a rare event, according to numerical simulations of Vorobyov \& Basu (2010b) and Vorobyov (2013) taking place in one out of ten models with similar characteristics. We do not think that the fragment survival is caused by a higher value of $\alpha$, since the aforementioned studies adopted a smaller value of $\alpha=5 \times 10^{-3}$. Instead, the present simulations suggest that the fragment survival can take place for a wide range of $\alpha$, meaning that this is a robust phenomenon.

One possible reason why model 6 demonstrated the fragment survival, while models 1 and 2 did not, is that the latter models experienced fragment ejection, losing $0.1 M_{\odot}$ and $0.25 M_{\odot}$ of the disk mass, respectively. This led to significant weakening of gravitational instability and virtual termination of disk fragmentation in the later evolution. On the other hand, model 6 lost only $0.02 M_{\odot}$ via ejection, which did not affect appreciably the strength of gravitational instability and fragmentation. The disk in model 6 continued to experience fragmentation even after $0.5 \mathrm{Myr}$ of evolution, which greatly increased the odds for fragment survival. It appears that the gravitational interaction between fragments in the disk is intrinsically a chaotic process, leading in some models to fragment ejection and in others to fragment survival.

Finally, the bottom row in Figure 1 presents the disk evolution in model 7 characterized by the non-zero massto-flux ratio $\mu_{\mathrm{B}}=3.33$. Other parameters in model 7 are identical to those of the non-magnetized model 1. The visual comparison of models 1 and 7 reveals that the frozenin magnetic field does not significantly change the disk propensity to fragment: the fragments are present in the disk during its early evolution. In the late evolution (after $t=0.5 \mathrm{Myr}$ ), the disk in the magnetized model seems to be more extended than its non-magnetized counterpart, but both show no signs of fragmentation. More accurate numerical simulations with non-ideal magnetohydrodynamical effects, such as ambipolar diffusion and magnetic braking, are planned for the near future.

We now analyze the efficiency of disk fragmentation in each model using a much higher time sampling than in Figure 1. Since we do not introduce sink particles to replace fragments in the disk in our Eulerian numerical code, it is very difficult to track the position and the fate of every fragment during the simulations. Therefore, we used the fragment detection algorithm described in detail in Vorobyov et al. (2013) to postprocess our results and calculate the number of fragments present in the disk at a given time. We discard fragments that are resolved by less than 10 grid cells ( 3 cells in each direction) since their identification on the numerical grid may be dubious. Figure 2 shows the number of fragments $N_{\mathrm{f}}$ calculated every $2000 \mathrm{yr}$ after the formation of the protostar in models $1-7$ (from top to bottom). Evidently, $N_{\mathrm{f}}$ varies significantly with time and from model to model. An increase in the number of fragments indicates recent fragmentation, and a decrease implies recent destruction/accretion/ejection of the fragments.

The $M_{\text {core }}=1.1 M_{\odot}$ model 1 demonstrates a strong

${ }^{6}$ We extended the run time to $1.0 \mathrm{Myr}$ and confirm that the fragments were still present in the disk. disk fragmentation activity in the early evolution. The mean number of fragments in a time period between $0.03 \mathrm{Myr}$ and 0.26 Myr is four. Episodically, the number of fragments may exceed 10 or drop to just a few. This means that the fragment formation and destruction mechanisms are constantly at play in the disk. At $t \approx 0.25 \mathrm{Myr}$ a massive fragment with some circumfragment material is ejected from the disk, reducing the total disk mass and weakening the gravitational instability. Part of the ejected material later falls back onto the disk triggering two isolated episodes of disk fragmentation at $t \approx 0.4 \mathrm{Myr}$ and $t \approx 0.7 \mathrm{Myr}$ but none of those fragments survive. The fragmentation activity in the $M_{\text {core }}=1.5 M_{\odot}$ model 2 is rather similar to that in model 1 except that there are no late-time fragmentation episodes in the disk, probably due to the fact that too much disk mass was lost during the ejection episode at $t \approx 0.32 \mathrm{Myr}$ and little fell back onto the disk. The $M_{\text {core }}=0.3 M_{\odot}$ model 3 shows just a few isolated disk fragmentation episodes with the number of fragments hardly exceeding one at a time. We do not show model 4 as it demonstrated no disk fragmentation.

The disk fragmentation activity in the $T_{\mathrm{bg}}=25 \mathrm{~K}$ model 5 is confined only to the initial 0.1 Myr of disk evolution. The likely explanation is that disk fragmentation in this model is driven by mass infall from the collapsing envelope, which is higher than in other models $\left(\dot{M}_{\text {infall }} \propto c_{\mathrm{s}}^{3}\right)$. As Figure 6 in Section 3.2 demonstrates, $\dot{M}_{\text {infall }}$ is maximal at the time of the protostar formation and quickly drops afterwards. The disk fragmentation process in the $\alpha=0.03$ model 6 continues for the whole duration of the simulation. We saw in Figure1 that several fragments managed to survive through almost 1.0 Myr of disk evolution. Figure 3 reveals that the number of fragments in the late evolution varies between one and just a few, meaning that actually only one fragment has evolved into a stable companion on a wide orbit. Other fragments form in the disk around the companion and in the spiral density waves connecting the companion with the primary disk rather than in the disk around the central star. This interesting effect will be studied in more detail in a followup paper.

Finally, the bottom panel in Figure2 presents the number of bursts vs. time in the magnetized model 7. Evidently, the frozen-in magnetic field with a mass-to-flux ratio of 3.33 does not appreciably change the disk fragmentation activity. The maximum number of fragments present in the disk at a specific time $\left(N_{\mathrm{fr}}^{\max }=8\right)$ is somewhat smaller in model 7 than in the corresponding nonmagnetized model $1\left(N_{\mathrm{fr}}^{\max }=12\right)$, but otherwise the time behaviour of $N_{\mathrm{fr}}$ is similar in both models.

\subsection{Accretion and infall rates}

In this section, we analyze the time behavior of mass accretion rates onto the star $(\dot{M})$ and infall rates onto the disk $\left(\dot{M}_{\text {infall }}\right)$ obtained in models $1-6$. We note that $\dot{M}$ is calculated at the position of the inner sink cell, $r_{\mathrm{cs}}=6 \mathrm{AU}$, while $\dot{M}_{\text {infall }}$ - at a distance of $2000 \mathrm{AU}$.

Figure 3 presents $\dot{M}$ (black solid lines) and $\dot{M}_{\text {infall }}$ (red dashed lines) vs. time in models $1-3$, which are characterized by different initial core masses $M_{\text {core }}$ as indicated in each panel. Other parameters in these models are similar. The time is counted from the begin- 
ning of numerical simulations (i.e., from the beginning of the core contraction). The formation of the central protostar in models 1-3 occurs around 0.15 Myr, $0.2 \mathrm{Myr}$, and 0.05 Myr, respectively. Evidently, accretion variability increases with increasing $M_{\text {core }}$. While the $M_{\text {core }}=0.3 M_{\odot}$ model 3 shows only an order of magnitude variations in $\dot{M}$ with just a few stronger fluctuations, the $M_{\text {core }}=1.1 M_{\odot}$ and $M_{\text {core }}=1.5 M_{\odot}$ models 1 and 2 are characterized by highly variable accretion spanning several orders of magnitude with peak values exceeding in magnitude $10^{-4} M_{\odot} \mathrm{yr}^{-1}$. These accretion bursts are caused by fragments spiralling into the central star due to the loss of angular momentum via gravitational interaction with spiral arms and other fragments in the disk (Vorobyov \& Basu 2005, 2006, 2010a). The animation of this process can be viewed at http://www.astro. uwo.ca/ basu/movies.html. We note that in model 2 one accretion burst at $\approx 0.52 \mathrm{Myr}$ is especially strong, exceeding in magnitude $10^{-3} M_{\odot} \mathrm{yr}^{-1}$. This burst is coeval with the fragment ejection event discussed in the previous section, a pair phenomenon that causes one fragment to fly into the intracluster medium and the other to fall onto the star (for more detail see Basu \& Vorobyov 2012).

We now discuss the reason why the time behaviour of mass accretion rates in models with increasing $M_{\text {core }}$ is so different. Figure 1 has already given us a hint - gravitational instability in models 1 and 2 is notably stronger than in model 3. This is not surprising considering that more massive cores are supposed to form more massive disks. To quantify the strength of gravitational instability in the disks of our models, we calculated global Fourier amplitudes using the following equation:

$$
C_{\mathrm{m}}(t)=\frac{1}{M_{\mathrm{d}}}\left|\int_{0}^{2 \pi} \int_{r_{\mathrm{sc}}}^{R_{\mathrm{d}}} \Sigma(r, \phi, t) e^{i m \phi} r d r d \phi\right|,
$$

where $M_{\mathrm{d}}$ is the disk mass, $R_{\mathrm{d}}$ is the disk's physical outer radius, and $m$ is the number of the spiral mode. When the disk surface density is axisymmetric, the amplitudes of all modes are equal to zero. When, say, $C_{\mathrm{m}}(t)=0.1$, the perturbation amplitude of spiral density waves in the disk is $10 \%$ that of the underlying axisymmetric density distribution.

The Fourier amplitudes of the first three spiral modes are presented in Figure 4 for models 1-3. Evidently, the $M_{\text {core }}=0.3 M_{\odot}$ model 3 is characterized by the lowest Fourier amplitudes - they hardly exceed $10 \%$ that of the underlying axisymmetric density distribution in the early evolution and quickly decline with time. The lower-order modes are generally higher in amplitude than the higherorder modes. On the other hand, the amplitudes of spiral modes in models 1 and 2 are appreciably higher than in model 3, reaching values as high as $60 \%$ that of the underlying axisymmetric distribution $\left(\log C_{\mathrm{m}} \approx-0.2\right)$ by the end of the embedded phase. In the later evolution, the Fourier amplitudes gradually decline with time, reflecting the overall disk stabilization due to continuing loss of disk material via accretion onto the star.

When comparing Figures 3 and 4 , we can notice a general correlation between Fourier amplitudes and variability in the mass accretion rates. The low- $M_{\text {core }}$ model 3 is characterized by both low Fourier amplitudes and low accretion variability. As $C_{\mathrm{m}}$ declines with time,

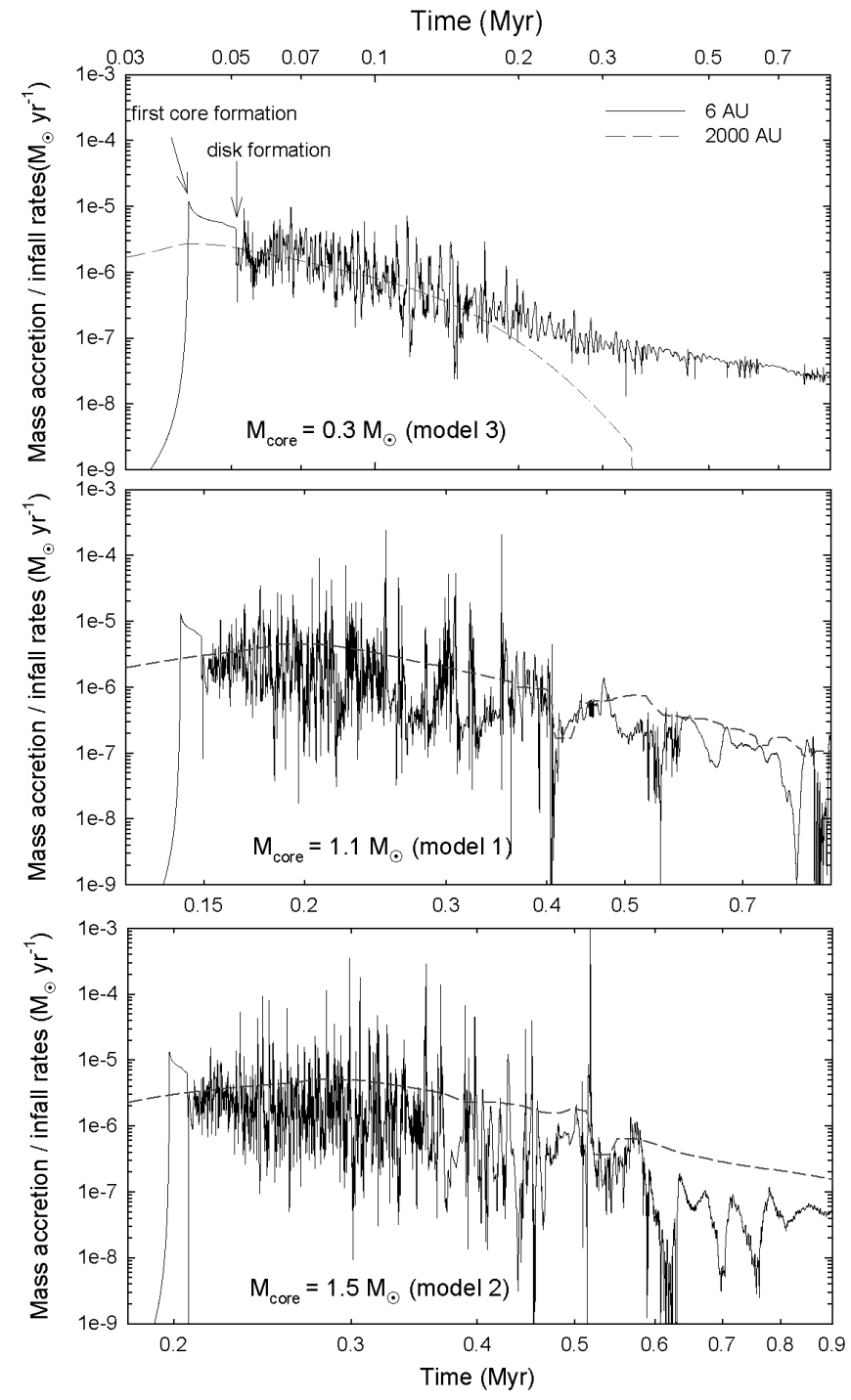

FIG. 3.- Mass accretion rates at $6 \mathrm{AU}$ (black solid lines) and envelope infall rates at $2000 \mathrm{AU}$ (red dashed lines) in models 1-3. The arrows mark the formation of the first hydrostatic core and the disk in model 1.

the variability in $\dot{M}$ diminishes. For $C_{\mathrm{m}} \lesssim 0.01$, the non-axisymmetric density perturbation in the form of spiral density waves is only $1 \%$ that of the underlying axisymmetric distribution, implying that the mass transport is now mostly controlled by viscous torques (Vorobyov \& Basu 2009). The latter drive the disk toward an axisymmetric state as evident in Figure 1 and the corresponding accretion rates show only lowamplitude flickering. On the other hand, Fourier amplitudes in the higher- $M_{\mathrm{c}}$ models 1 and 2 are greater than in model 3 and the variability in the corresponding accretion rates is notably higher. Fourier amplitudes comparable to or greater than $10 \%$ imply the presence of strong non-axysimmetry in the disk in the form of both spiral waves and fragments. It is important to note that Fourier amplitudes are highly variable ${ }^{7}$ due to the dynamical interaction of spiral density waves and fragments in the disk. A combination of two effects: that of

7 In fact, the time variability in $C_{m}$ is even higher but we had to smooth Fourier amplitudes somewhat to make them discernible in the figure. 

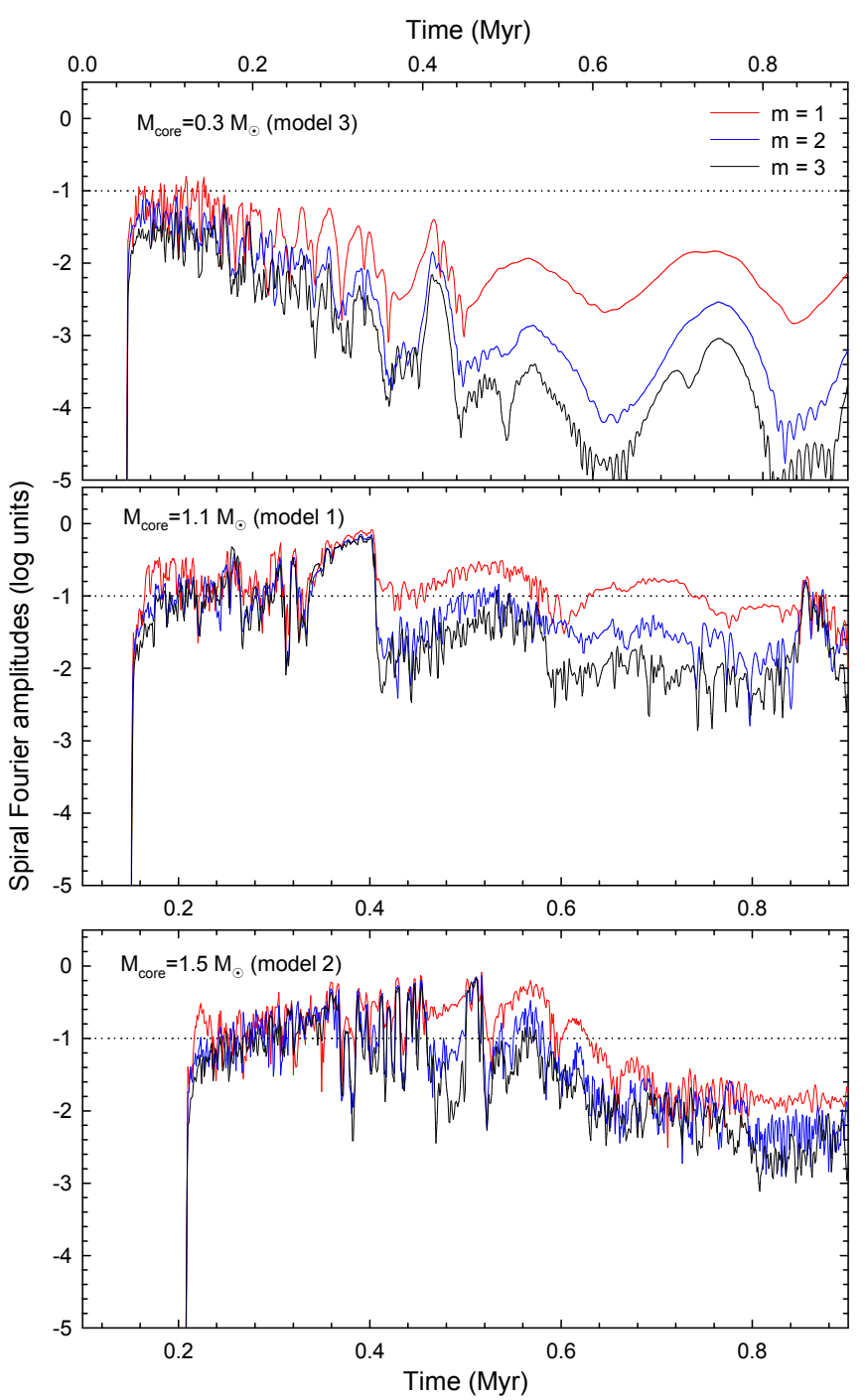

Fig. 4.- Global Fourier amplitudes of the first three spiral modes $m=1-3$ in models $1-3$. The horizontal dotted lines mark the boundary above which spiral waves have perturbation amplitudes greater than $10 \%$ that of the underlying axisymmetric density distribution.

the nonlinear interaction between different spiral modes and fragments in the disk and that of the fragments spiralling down onto the star produces variable accretion with episodic bursts.

We now proceed with analyzing the effect of different values of $\beta, T_{\mathrm{bg}}, \alpha$-parameter on the variability of $\dot{M}$. Figure 5 presents the mass accretion and infall rates in model 4 characterized by $\beta=0.14 \%$, a factor of six lower than in model 1. The other parameters in both models are identical. Evidently, the mass accretion rate in the low- $\beta$ model 4 is characterized by low-amplitude flickering and complete absence of bursts, while the high- $\beta$ model 1 shows strong accretion variability with multiple bursts, some exceeding in magnitude $10^{-4} M_{\odot} \mathrm{yr}^{-1}$. As was demonstrated in Vorobyov (2010), pre-stellar cores with higher $\beta$ tend to form more massive and extended disks than cores with lower $\beta$, which can be understood on the basis of simple centrifugal radius arguments. Massive and extended disks are more gravitationally unstable and prone to fragmentation than light and compact ones, explaining the aforementioned tendency in $\dot{M}$.

As a next step, we describe the effect of a higher back-

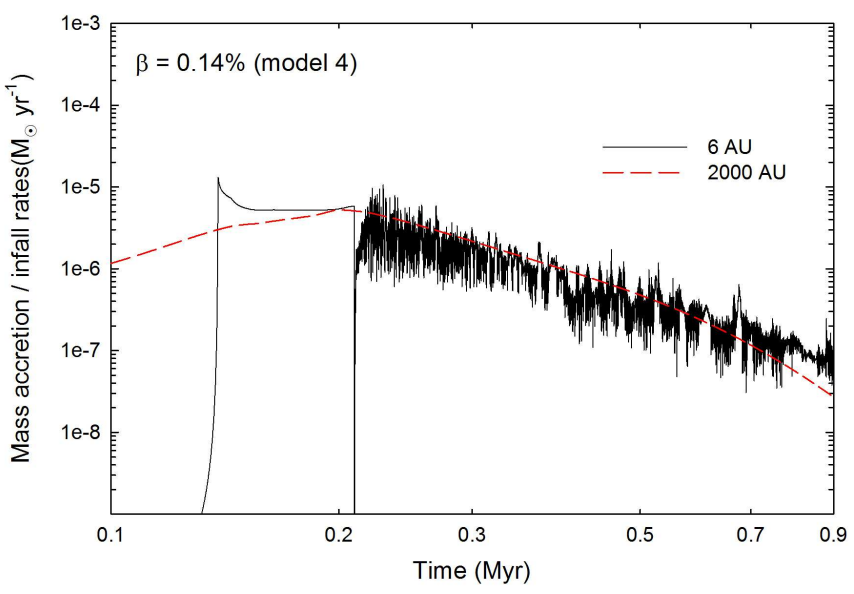

FIG. 5.- Mass accretion rates at $6 \mathrm{AU}$ (black solid lines) and envelope infall rates at $2000 \mathrm{AU}$ (red dashed lines) in models 4.

ground temperature $T_{\mathrm{bg}}$ on the time behaviour of mass accretion rates, thus mimicking a higher heating rate coming from the external environment. The top panel in Figure 6 presents $\dot{M}$ and $\dot{M}_{\text {infall }}$ vs. time in model 5 characterized by $T_{\mathrm{bg}}=25 \mathrm{~K}$, which is 2.5 times higher than the corresponding value in model 1 . The other parameters are identical in both models. The increase in $T_{\text {bg }}$ reduces notably the accretion variability. This effect is explained by the fact that a higher background temperature raises the overall disk temperature and reduces the strength of disk gravitational instability. Nevertheless, the $T_{\mathrm{bg}}=25 \mathrm{~K}$ model 5 exhibits several bursts with $\dot{M} \approx 10^{-4} M_{\odot} \mathrm{yr}^{-1}$.

The middle panel in Figure 6 presents $\dot{M}$ and $\dot{M}_{\text {infall }}$ vs. time in model 6 , the parameters of which are similar to those of model 1 except that the $\alpha$-parameter is set now to 0.03 , a factor of six higher than in model 1 . Evidently, the increase in $\alpha$ acts to reduce the variability in $\dot{M}$, which now features only order-of-magnitude variations. Nevertheless, there are two well-pronounced accretion bursts with $\dot{M}$ approaching $10^{-4} M_{\odot} \mathrm{yr}^{-1}$. The notable reduction in the burst frequency in model 6 as compared to the low- $\alpha$ model 1 can be attributed to increased viscous mass transport through the disk, which reduces both the disk mass and the strength of gravitational instability (Vorobyov \& Basu 2010a). Viscosity also tends to smooth out local non-axisymmetric density enhancements (Vorobyov \& Basu 2009), thus reducing the accretion variability caused by spiral density waves.

The last model in this study takes into account the effect of frozen-in magnetic fields. The bottom panel in Figure 6 presents the mass accretion and infall rates in model 7 which has the same parameters as the nonmagnetic model 1 except that the mass-to-flux ratio is set to $\mu_{\mathrm{B}}=3.33$. Evidently, the magnetized model 7 exhibits several strong accretion bursts with a rate $\geq$ $10^{-4} M_{\odot} \mathrm{yr}^{-1}$, indicating that the frozen-in magnetic field does not suppress the burst phenomenon.

Finally, we briefly discuss the time behaviour of infall rates in our models and a possible link between $\dot{M}_{\text {infall }}$ and the burst phenomenon. Figures 3 , 5, and 6] demonstrate that $\dot{M}_{\text {infall }}$ steadily increases from $\approx$ $10^{-6} M_{\odot} \mathrm{yr}^{-1}$ to $(5-10) \times 10^{-6} M_{\odot} \mathrm{yr}^{-1}$ during the early evolution and then gradually declines below $10^{-7} M_{\odot} \mathrm{yr}^{-1}$, reflecting the overall depletion of mass 

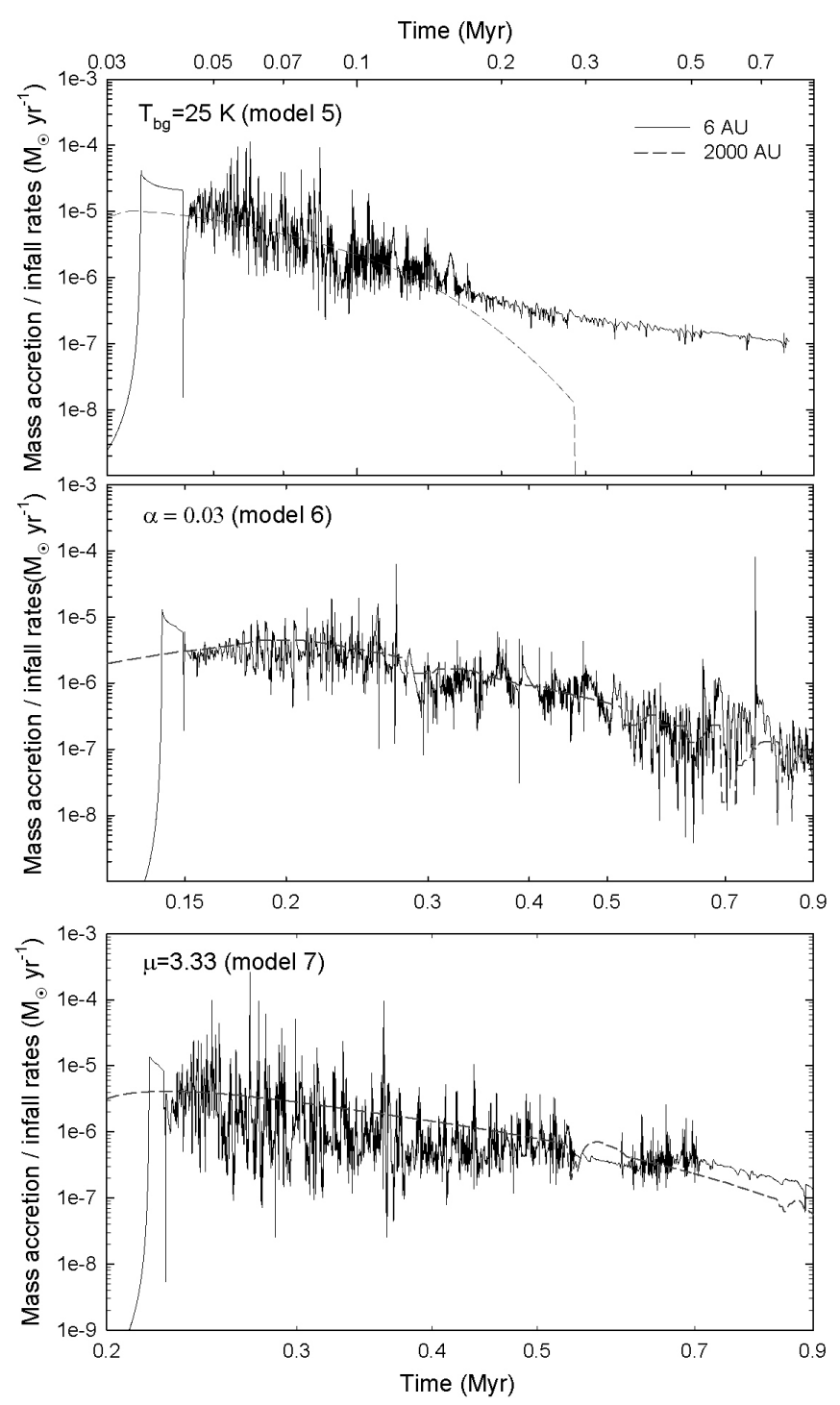

FiG. 6.- Mass accretion rates at $6 \mathrm{AU}(\dot{M}$, black solid lines) and envelope infall rates at $2000 \mathrm{AU}\left(\dot{M}_{\text {infall }}\right.$, red dashed lines) in models 5,6 , and 7 (from top to bottom).

in the parental cores. Order-of-magnitude variations of $\dot{M}_{\text {infall }}$ in the late evolution of models 1,2 , and 6 are caused by the disturbing influence of fragments that were ejected from the disk. A visual inspection of $\dot{M}$ and $\dot{M}_{\text {infall }}$ in Figures 3, 5, and 6 reveals that the amplitude of variations in the accretion rate generally correlates with the infall rate, though with some notable exceptions. More specifically, for $10^{-6} M_{\odot} \mathrm{yr}^{-1} \leq \dot{M}_{\text {infall }} \leq$ $10^{-5} M_{\odot} \mathrm{yr}^{-1}$ both the large-scale accretion variability and the bursts are present. For $10^{-7} M_{\odot} \mathrm{yr}^{-1} \leq$ $\dot{M}_{\text {infall }} \leq 10^{-6} M_{\odot} \mathrm{yr}^{-1}$ some variability in accretion rates is still present but the bursts are mostly gone, except for model 6 showing one energetic burst in the late evolution. For $\dot{M}_{\text {infall }} \leq 10^{-7} M_{\odot} \mathrm{yr}^{-1}$ the accretion variability diminishes and no bursts are seen. The character of accretion for various infall rates is summarized in Table 2 ,

Model 4, however, stands apart and shows little accretion variability and no bursts even though the infall rates are greater than $10^{-6} M_{\odot} \mathrm{yr}^{-1}$ during the early evolu-

TABLE 2

INFALL RATES AND THE CHARACTER OF ACCRETION

\begin{tabular}{ccc}
\hline \hline $\begin{array}{c}M_{\text {infall }} \\
\left(M_{\odot} \mathrm{yr}^{-1}\right)\end{array}$ & $\begin{array}{c}\text { Variability } \\
\text { (orders of mag.) }\end{array}$ & Bursts \\
\hline $10^{-5}-10^{-6}$ & $2-3$ & multiple \\
$10^{-6}-10^{-7}$ & $\sim 1$ & occasional \\
$<10^{-7}$ & $<1$ & no \\
\hline
\end{tabular}

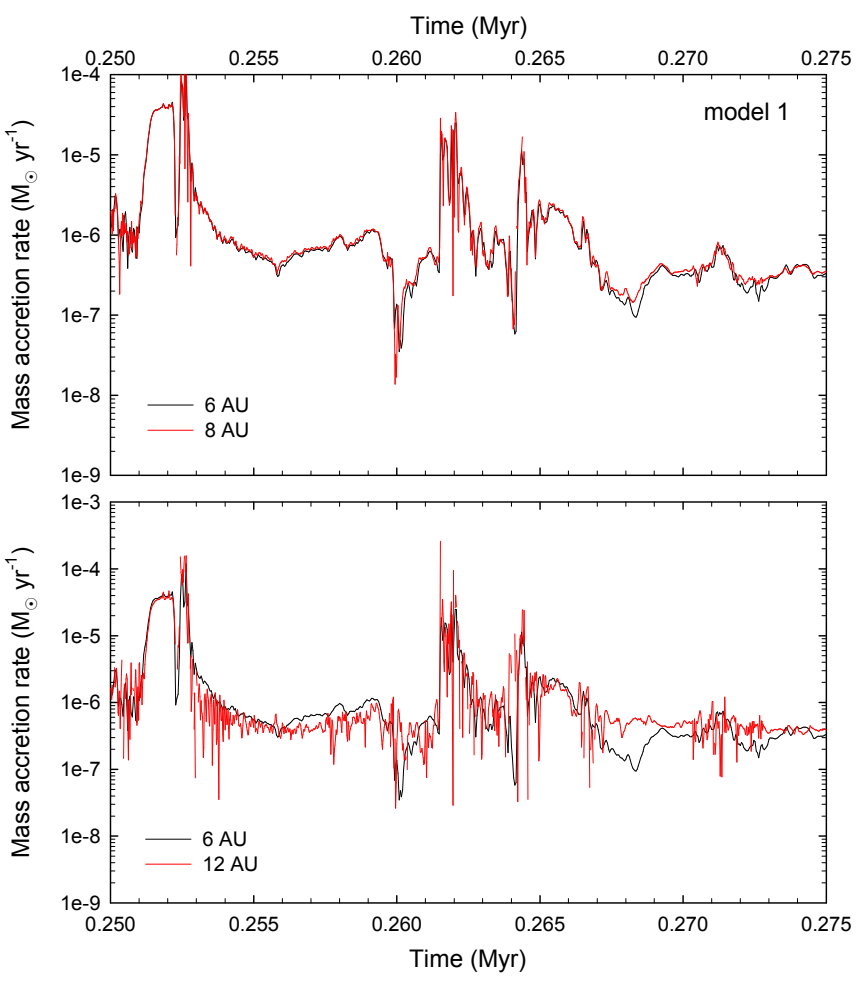

FIG. 7.- Comparison of the mass accretion rates at $6 \mathrm{AU}$ and $8 \mathrm{AU}$ (top panel) and $6 \mathrm{AU}$ and $12 \mathrm{AU}$ (bottom panel) during a short period of evolution in model 1.

tion. Due to its low angular momentum, the $\beta=0.14 \%$ model 4 starts forming the disk considerable later than other models. As a result, the disk mass and radius are not sufficiently large for gravitational fragmentation to take place (see also Fig 1). High infall rates are therefore a necessary but not sufficient condition for the development of the burst phenomenon.

To summarize this section, our numerical simulations with the updated code confirmed the findings reported earlier in Vorobyov \& Basu (2010a), namely that an increase in the initial core mass $M_{\text {core }}$ and/or the ratio of rotational to gravitational energy $\beta$ acts to increase the amplitude of accretion variability and the number of accretion bursts. An increase in the $\alpha$-parameter and $T_{\mathrm{bg}}$ is found to have the opposite effect - the accretion variability and bursts diminish but do not cease to exist, at least for reasonable values of $\alpha$ and $T_{\mathrm{bg}}$. This means that accretion variability and bursts are a robust phenomenon, weakly sensitive to (modest) variations in, e.g., dust opacities, turbulent viscosity, and stellar radiation.

\subsection{The effect of the inner boundary condition}

In our models, the mass accretion rate $\dot{M}$ is calculated at the position of the inner sink cell, $r_{\mathrm{sc}}=6 \mathrm{AU}$. Two questions arise in this context: how much $\dot{M}$ is sensitive 
both to the choice of $r_{\mathrm{sc}}$ and to the imposed free outflow boundary condition through the sink cell. We cannot compute the disk dynamics at sub-AU scales because of a strict Courant time step limitation imposed on our explicit Eulerian code. However, we performed several test runs with $r_{\mathrm{sc}}$ varied by a factor of 2 and found little effect on the accretion burst phenomenon.

This result can be easily understood by the following reason. Fragments form in the disk at a radial distance of at least several tens AU from the sink cell where conditions become favourable for gravitational fragmentation (e.g. Meru \& Bate 2012). At such distances, the influence of the inner boundary on the disk fragmentation process is negligible. Furthermore, the typical size of fragments approaching the inner sink cell is comparable to the size of the sink cell itself. Therefore, varying $r_{\mathrm{sc}}$ by a factor of 2 does not make much difference - the fragment will sooner or later pass through the sink cell. On the other hand, as fragments approach the star, they must be inevitably stretched out due to tidal torques. How much of the fragment material finally reaches the star is an open question and requires a focused investigation (see e.g. Cha \& Nayakshin 2011; Nayakshin \& Lodato 2012). The effect of a sudden mass deposition onto the inner disk $(\leq 10 \mathrm{AU})$, as if by infall of a fragment migrating through the disk onto the star, has recently been investigated by Ohtani et al. (2014). It was found that such an event can lead to the FU-Orionislike eruption due to triggering of the magneto-rotational instability (MRI) at sub-AU scales. This means that either directly, by deposition of material onto the star, or indirectly, by triggering the MRI in the inner disk, the inward migration of fragments will likely produce luminosity outbursts.

To evaluate the possible influence of the free outflow boundary condition on the mass accretion rate through the sink cell, we calculated the mass transport rates at a few AU away from the sink cell. Figure 7 presents the mass accretion rates through the sink cell at 6 AU $(\dot{M}$, black lines), as well as the mass transport rates through the disk at $8 \mathrm{AU}\left(\dot{M}_{8}\right.$, red line $)$ and $12 \mathrm{AU}\left(\dot{M}_{12}\right.$, red lines). A narrow time interval of $2 \times 10^{4} \mathrm{yr}$ in model 1 is chosen to focus on a time period featuring both the bursts and quiescent accretion. Evidently, $\dot{M}_{8}$ is very similar to $\dot{M}$ except for a few instances when notable deviations are visible. On the other hand, $\dot{M}_{12}$ demonstrates larger deviations from $\dot{M}$, but retains the main qualitative features of $\dot{M}$ such as accretion bursts. Both $\dot{M}_{8}$ and $\dot{M}_{12}$ exhibit more variability than $\dot{M}$, most likely due to the fact that the inner boundary allows for matter to flow into the sink cell but not out of it, thus somewhat artificially damping the time variations.

\section{CHARACTERISTICS OF LUMINOSITY BURSTS}

In this section, we analyze the characteristics of luminosity bursts obtained in our models and compared them with the available statistics on FU-Orionis-type eruptions (FUors) taken from the recent review paper by Audard et al. (2014). In order to distinguish the bursts from regular (order-of-magnitude) variability in our models we have to make several assumptions. First, we assume that the total luminosity $L_{*}$ during the burst should be comparable to that of FUors. The latter are usually characterized by an increase in brightness by at least a factor of 3-4 (in stellar magnitudes) as compared to the pre-burst, quiescent phase. Therefore, we stipulate that the luminosity increase during the burst should be at least 16 times $(\sim 3 \mathrm{mag})$ that of the pre-burst phase.

Calculating the luminosity in the pre-burst phase turned out to be not an easy task due to a highly changeable nature of accretion. We do this by defining the socalled background luminosity $L_{\mathrm{bg}}$, which comprises the stellar photospheric luminosity $L_{*, \text { ph }}$ and the mean accretion luminosity $\left\langle L_{*}\right.$,accr $\rangle$. The former is provided by a stellar evolution code (see Section 2), while the latter is found as

$$
\left\langle L_{*, \mathrm{accr}}\right\rangle=\frac{G M_{*}\langle\dot{M}\rangle}{2 R_{*}},
$$

where $\langle\dot{M}\rangle$ is the mean accretion rate calculated using a running average of the instantaneous accretion rates $\dot{M}$ over a time period of $10^{4} \mathrm{yr}$. When doing the average, we filtered out values that are greater than $5 \times 10^{-6} M_{\odot} \mathrm{yr}^{-1}$ by the reason that they may already represent a burst in its rising or fading phase.

The red and black lines in Figure 8 present the total luminosity $L_{*}$ and background luminosity $L_{\mathrm{bg}}$ in models $1,2,5$, and 7 . We left out models that showed too few bursts to be statistically meaningful. In general, $L_{*}$ is highly variable in the early evolution, reflecting the corresponding variations in the mass accretion rate. On the other hand, $L_{\mathrm{bg}}$ shows much less variability and describes well the minimal luminosity in each model. The blue lines mark the values that are 16 times greater than the background luminosity at a given time, representing therefore the 3-magnitude cutoff above which a surge in luminosity may be classified as a FUor. In principle, these relatively modest bursts can be confused with the so-called EXors named after its prototype EX Lupi (see e.g. Audard et al. 2014). Therefore, with the blue line we also plot the 4-magnitude cutoff (39 times greater than $L_{\mathrm{bg}}$ ) in order to analyze the statistics of more energetic bursts, which are more likely to represent bona fide FUors.

Evidently, models 1 and 2 are characterized by the largest number of strong FUor-type bursts, amounting to 10 and more per model ${ }^{8}$. The higher- $T_{\mathrm{bg}}$ model 5 has only a few bursts above the 4-magnitude cutoff. At the same time, model 7 demonstrates several strong bursts despite the presence of frozen-in magnetic field with a mass-to-flux ratio $\mu_{\mathrm{B}}=3.33$, indicating that FUors can occur in magnetized disks as well.

In the following text, we analyze the main characteristics of the luminosity bursts obtained in our models in order to compare our predictions with observations. Figure 9 presents the duration of the burst $t_{\mathrm{bst}}$ (left column), the accreted mass during the burst $M_{\text {accr }}$ (middle column), and the peak luminosity during the burst $L_{\mathrm{bst}}^{\max }$ (right column) in models 1, 2, 5 and 7 (from top row to bottom one). Only bursts with a 4-mag cutoff are shown. The $x$-axis shows the ordinary number of the burst arranged along the line of the burst occurrence. Evidently, the burst duration stays mostly in the 10-100 yr limit with little dependence either on time or particular model.

\footnotetext{
8 Some of the bursts are closely packed and cannot be resolved in the figure (see Section 6).
} 

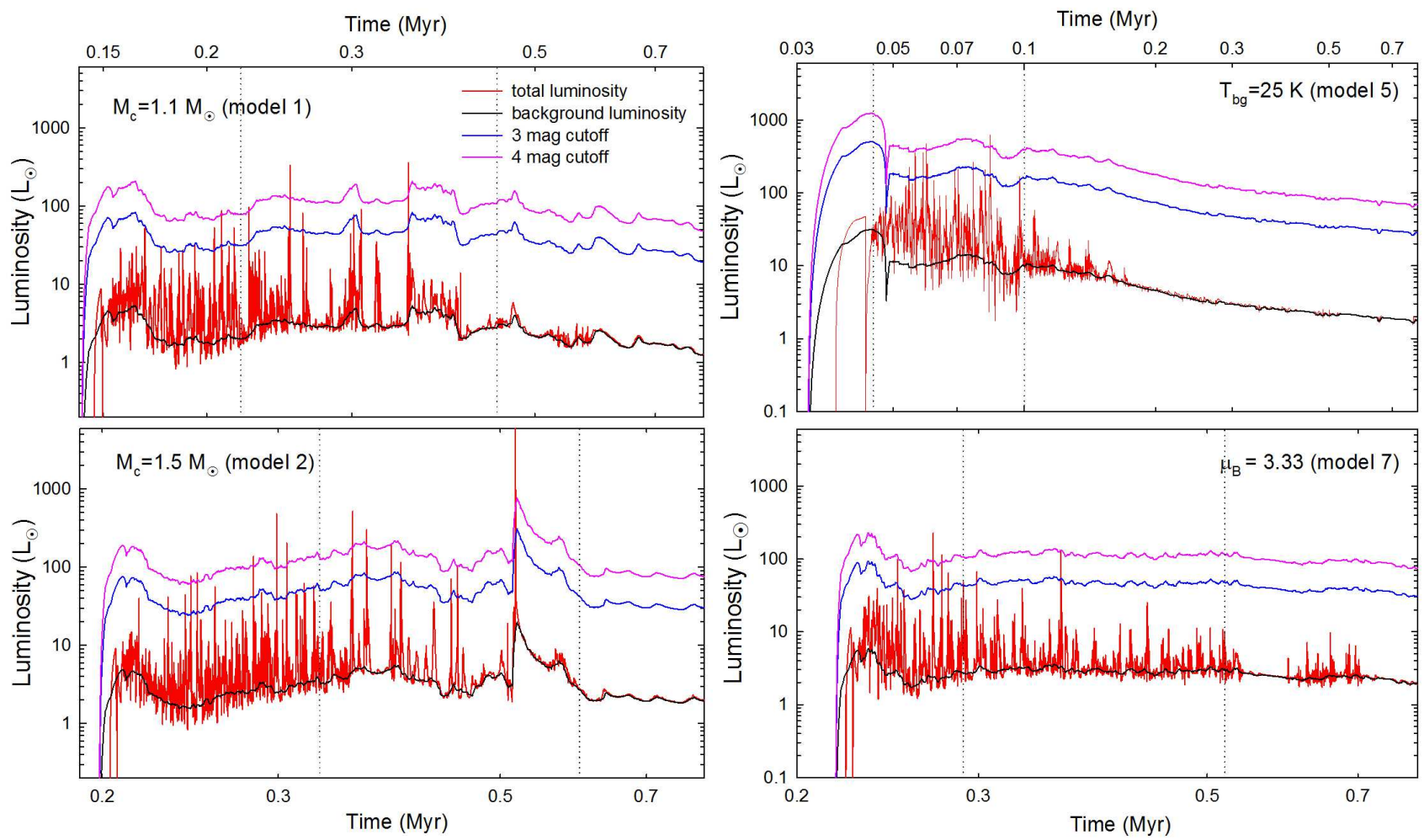

FIG. 8.- Red lines: total (accretion plus photospheric) luminosity vs. time in model 1 (top left), model 2 (bottom-left), model 5 (top-right), and model 7 (bottom-right). The black lines provide the background luminosity comprising the photospheric luminosity plus accretion luminosity arising from accretion with a rate $\leq 5 \times 10^{-6} M_{\odot} \mathrm{yr}^{-1}$. The blue and pink lines mark the 3-magnitude and 4magnitude cutoffs above which a surge in luminosity is considered to be an FU-Orionis-type outburst. The vertical dotted lines mark the Class 0/I boundary (left lines) and Class I/II boundary (right lines). See the text for more details.

These values are in good agreement with the measured or inferred duration of FUors (see Table 2 below and table 1 in Audard et al. 2014). The accreted mass during the bursts ranges from 1.0 to 75 Jupiter masses, covering the full mass range of giant planets and brown dwarfs and reflecting the mass range of fragments forming in the disk (Vorobyov et al. 2013). The peak luminosities of most bursts span a range between $75 L_{\odot}$ and $600 L_{\odot}$, with a few notable exceptions in model 2 reaching values in excess of $3000 L_{\odot}$. These most luminous (and closelypacked) bursts occur during the fragment ejection event discussed in Section 3.1 and are the result of the conservation of angular momentum, causing one fragment to fly out of the disk and the other fragment to fall onto the star due catastrophic loss of angular momentum during the close encounter. The fact that there are three closelypacked bursts instead of just one is explained by tidal destruction of the infalling fragment (see Section [6).

The summary of various characteristics of the bursts obtained in our modeling are provided in Table 3. More specifically, the second column provides the total number of bursts $N_{\text {bst }}$ and the number of bursts in the deeply embedded Class 0 phase (in parentheses, see Section 5), the third column is the fraction of stellar mass accreted during the bursts $M_{\mathrm{bst}}^{\mathrm{tot}}$, and the fourth column is the fraction of total disk lifetime spent in the burst phase $t_{\mathrm{bst}}^{\mathrm{tot}}$. The other columns (from 5th to 9th) present the maximum, minimum and mean luminosities of the bursts ( $L_{\max }, L_{\min }$ and $\left.L_{\text {mean }}\right)$, the maximum, minimum and mean accretion rates during the bursts $\left(\dot{M}_{\max }, \dot{M}_{\min }\right.$ and $\dot{M}_{\text {mean }}$ ), the maximum, minimum and mean burst durations $\left(t_{\mathrm{bst}}^{\max }, t_{\mathrm{bst}}^{\min }\right.$ and $\left.t_{\mathrm{bst}}^{\text {mean }}\right)$, the maximum, mini-
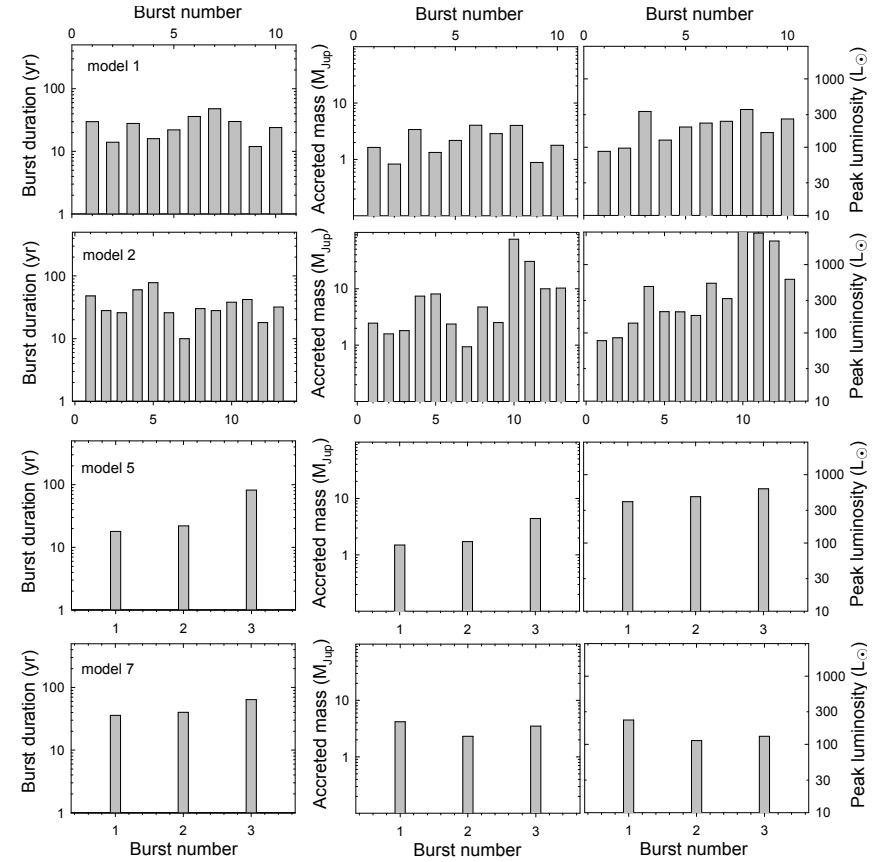

FIG. 9.- Burst characteristics in model 1 (top row), 2 (middle row) and 5 (top row). Columns (from left to right) present the duration of the bursts $t_{\mathrm{bst}}$ (in yr), the accreted mass during the bursts $M_{\text {accr }}$ (in $M_{\text {Jup }}$ ), and the peak luminosity during the bursts $L_{\mathrm{bst}}^{\max }\left(\right.$ in $\left.L_{\odot}\right)$.

mum and mean duration of the quiescent phase between the $\operatorname{bursts}^{9}\left(t_{\mathrm{qst}}^{\max }, t_{\mathrm{qst}}^{\min }\right.$ and $\left.t_{\mathrm{qst}}^{\text {mean }}\right)$, and the maximum

${ }^{9}$ We calculated these quantities by making no distinction be- 
and mean accreted mass during the bursts $\left(M_{\text {accr }}^{\max }\right.$ and $\left.M_{\text {accr }}^{\text {mean }}\right)$. The mean values were found by arithmetically averaging over all bursts in a particular model. The known characteristics of FUors are provided in the bottom line and are taken from table 1 of Audard et al. (2014).

Our models seem to reproduce the main properties of FUors rather well. When averaged over all models, the mean luminosity during the burst in our models is $312 L_{\odot}$, about a factor of 1.5 greater than that of FUors. The agreement becomes even better after taking out rare outliers such as very energetic bursts in model 2 . The averaged burst duration is $39 \mathrm{yr}$, again almost a factor of 2 greater than that of FUors, but this may be simply due to the fact that many FUors are still found in the active phase. The duration of the quiescent phase also seems to be in agreement with the recently estimated lower limit of 5-10 kyr using mid-infrared photometry (Scholz et al. 2013). There is however some disagreement which may be of physical nature. For instance, our model accretion rates agree well with the mean inferred mass accretion rates in FUors, but fail to explain low- $\dot{M}$ objects such as HBC 722 with $\dot{M} \sim 10^{-6} M_{\odot} \mathrm{yr}^{-1}$ (Audard et al. 2014). Indeed, for the minimum accreted mass of 1.0 Jupiter and the maximum duration of the burst of $100 \mathrm{yr}$, the resulting minimum accretion rates in our models are supposed to be around $10^{-5} M_{\odot} \mathrm{yr}^{-1}$, implying that FUors like HBC 722 may be driven by mechanisms other than disk fragmentation.

\section{EMBEDDED VS. OPTICALLY VISIBLE BURSTS}

According to Quanz et al. (2007), FUors can be classified in two categories, depending on whether silicate features at $10 \mu \mathrm{m}$ are seen in absorption or emission. FUors with silicate in absorption are likely still embedded in parental envelopes, whereas FUors with silicates in emission are likely more evolved and having (partially) depleted envelopes. Out of 21 observed FUors in the Quanz et al. sample, 12 have silicates in absorbtion and 7 in emission, with 2 objects having a flat spectrum (making their classification dubious). This simple analysis suggests that most FUors are rather young objects, possessing sizeable envelopes.

A similar conclusion can be made using data summarized in the recent review on episodic accretion in young protostars by Audard et al. (2014). Two prominent objects, FU Ori itself and V1515, are often considered as most evolved FUors having little-to-no envelope, suggested by weak far-infrared/submillimeter continuum beyond $100 \mu \mathrm{m}$ in their spectral energy distributions ${ }^{10}$. According to table 1 in Audard et al., these two objects have the optical extinction $A_{V}$ lying in the 1.5-3.2 range. On the other hand, young FUors with silicate in absorption have a minimum extinction of $A_{V}=4.2$ (Quanz et al. 2007). We therefore set $A_{V}=4.0$ as a tentative boundary between embedded and optically visible FUors. In the Audard et al.'s sample, 15 FUors are characterized by $A_{V}>4.0$ and only 6 FUors have $A_{V}<4.0$. Of course, this simple analysis may somewhat be affected

\footnotetext{
tween isolated and clustered bursts, see Section 6

10 Even in this case, near-infrared interferometry shows that, e.g., V1515 may have an undetected contribution from the envelope (Millan-Gabet et al. 2006).
}

by inclination: highly inclined FUors may appear embedded, whereas in reality they are not. Nevertheless, all above arguments taken together indicate that many (if not most) FUors are young, embedded objects rather than older, Class II stars.

To classify the bursts in our models, we use the remaining mass in the envelope to define the boundary between the embedded and optically visible phases. Namely, we assume that the optically visible Class II begins when less than $10 \%$ of the initial core mass is left in the envelope. The boundary between the deeply embedded Class 0 phase and the partly embedded Class I phase is defined as the time when $50 \%$ of the initial core mass is left in the envelope. Our adopted classification scheme is based on physical properties of a young stellar object, such as envelope and disk masses (e.g., Robitaille et al. 2006; Dunham et al. 2010, 2014), rather than on observational signatures, such as submillimeter luminosities or effective temperatures (e.g. André et al. 1993; Chen et al. 1995). Classifications relying upon physical properties are usually referred in the literature as "stages", whereas those using observational signatures are called "classes". For simplicity here we use the term "class" to refer to both the physical stages and observational classes. Our adopted definition of physical stages was extensively investigated in Dunham et al. (2010). They found that there is not always a one-to-one correspondence between physical stage defined by the envelope mass and observational class defined by the submillimeter luminosity or effective temperature due to the effects of geometry and extinction. In reality the exact point at which to set the class boundaries is somewhat uncertain, which could shift the duration of the embedded phase in our models by a factor of order unity in either direction. We disentangle the disk and infalling envelope on our numerical grid using the algorithm described in Vorobyov (2011), which is based on the disk-to-envelope transition density of $\Sigma_{\text {crit }}=0.5 \mathrm{~g} \mathrm{~cm}^{-2}$ and the velocity field in the infalling envelope. Varying the value of $\Sigma_{\text {crit }}$ by a factor of 5 results in changes of the estimated onset time of different phases by only a few per cent.

The vertical dotted lines in Figure 8 mark the Class 0/I boundary (left lines) and the Class I/II boundary (right lines). Evidently, most bursts in our models occur in the partly-embedded Class I phase. For instance, model 1 has nine strong bursts (above 4-mag cutoff) taking place in the Class I phase, and only one in the Class 0 phase, while model 2 has only five strong bursts out of 13 occurring in the Class 0 phase. The values in parentheses in the second column of Table 3 provide the number of bursts (out of the total number) occurring in the Class 0 phase. Out of the total 29 strong bursts, only 8 occurred in the deeply embedded Class 0 phase and 21 in the partly-embedded Class I phase. As similar though less pronounced tendency is found for less energetic bursts (3-mag cutoff), except for the magnetized model 7, in which most bursts occur in the early Class 0 phase.

Our models notably lack bursts taking place in the optically visible Class II phase. This is not surprising since most fragments form in the early evolution, which is characterized by most massive and gravitationally unstable disks (see Figs. 1 and 2). This phase is also less favourable for the survival of fragments owing to strong gravitational and tidal torques which tend to drive frag- 
TABLE 3

Characteristics OF LUminosity Bursts

\begin{tabular}{|c|c|c|c|c|c|c|c|c|}
\hline Model & $N_{\text {bst }}$ & $\begin{array}{c}M_{\mathrm{bst}}^{\mathrm{tot}} \\
(\%)\end{array}$ & $\begin{array}{l}t_{\mathrm{bst}}^{\mathrm{tot}} \\
(\%)\end{array}$ & $\begin{array}{c}L_{\max } / L_{\min } / L_{\text {mean }} \\
\left(L_{\odot}\right)\end{array}$ & $\begin{array}{c}M_{\max } / \dot{M}_{\min } / \dot{M}_{\text {mean }} \\
\left(10^{-4} M_{\odot} \mathrm{yr}^{-1}\right)\end{array}$ & $\begin{array}{c}t_{\mathrm{bst}}^{\max } / t_{\mathrm{bst}}^{\min } / t_{\mathrm{bst}}^{\operatorname{mean}} \\
(\mathrm{yr})\end{array}$ & $\begin{array}{c}t_{\mathrm{qst}}^{\max } / t_{\mathrm{qst}}^{\min } / t_{\mathrm{qst}}^{\text {mean }} \\
\left(10^{4} \mathrm{yr}\right)\end{array}$ & $\begin{array}{c}M_{\text {accr }}^{\max } / M_{\text {accr }}^{\text {mean }} \\
\left(M_{\text {Jup }}\right) \\
\end{array}$ \\
\hline \multicolumn{9}{|c|}{ 4-mag cutoff } \\
\hline 1 & $10(1)$ & 3.8 & 0.035 & $357 / 87 / 208$ & $2.4 / 0.78 / 1.4$ & $48 / 12 / 25$ & $10 / 1.6 / 4.7$ & $4.0 / 2.3$ \\
\hline 2 & $13(5)$ & 18.6 & 0.06 & $3042 / 77 / 846$ & $20 / 0.8 / 5.3$ & $78 / 10 / 36$ & $15 / 0.36 / 4.4$ & $75 / 12$ \\
\hline 5 & $3(0)$ & 1.3 & 0.02 & $620 / 403 / 500$ & $1.2 / 0.92 / 1.0$ & $82 / 18 / 41$ & - & $4.4 / 2.5$ \\
\hline 7 & $3(2)$ & 2.0 & 0.04 & $227 / 115 / 157$ & $2.6 / 0.9 / 1.5$ & $64 / 36 / 47$ & - & $4.1 / 3.3$ \\
\hline \multicolumn{9}{|c|}{ 3-mag cutoff } \\
\hline 1 & $21(9)$ & 6.4 & 0.086 & $357 / 28 / 110$ & $2.4 / 0.25 / 0.81$ & $120 / 2 / 31$ & $4.3 / 0.005 / 1.0$ & $9.0 / 1.9$ \\
\hline 2 & $42(15)$ & 24.7 & 0.24 & $3042 / 25 / 300$ & $20 / 0.14 / 2.1$ & $320 / 2 / 47$ & $6.4 / 0.002 / 0.8$ & $75 / 6$ \\
\hline 5 & $15(0)$ & 5.2 & 0.09 & $620 / 163 / 290$ & $1.2 / 0.21 / 0.64$ & $132 / 14 / 50$ & $1.4 / 0.004 / 0.33$ & $5.8 / 2.4$ \\
\hline 7 & $12(8)$ & 4.4 & 0.1 & $227 / 37 / 81$ & $2.6 / 0.37 / 0.79$ & $90 / 6 / 33$ & $6.2 / 0.002 / 1.1$ & $6.7 / 1.9$ \\
\hline \multicolumn{9}{|c|}{$\begin{array}{c}\text { FUors } \\
\text { (observations) }\end{array}$} \\
\hline & 26 & - & - & $525 / 10 / 200$ & $10 / 0.01 / 1.9$ & $80 / 4 / 20$ & - & - \\
\hline
\end{tabular}

ments onto the star or destroy them. On the other hand, fragments that happen to survive through the embedded phase are more likely to form stable companions, rather than to migrate onto the star and trigger a burst.

There are however exceptions. The middle panel in Figure 6 shows the mass accretion rate in model 6 . The Class II phase in this model starts at $t=0.47 \mathrm{Myr}$ after the onset of gravitational collapse. A strong accretion burst at $t=0.763 \mathrm{Myr}$, corresponding to a luminosity outburst of $373 L_{\odot}$, occurs well into the optically visible Class II phase.

Model 6 is unique among other models in the sense that it reveals the formation of a wide-orbit companion on a quasi-stable orbit. Figure 10 presents the gas surface density in model 6 in the inner box of $1400 \times 1400$ AU just before the luminosity outburst and immediately after it. Evidently, the survived companion possesses a circumfragment disk, which is sufficiently massive to experience episodic fragmentation. Indeed, the mass of the companion and its disk are $57 M_{\text {Jup }}$ and $21 M_{\text {Jup }}$, making the disk to central object mass ratio equal to $\xi \approx 0.38$. Systems with $\xi \geq 0.1$ are likely to be unstable to fragmentation (Vorobyov \& Basu 2010a). For comparison, the masses of the central star and its circumstellar disk at this time are $0.7 M_{\odot}$ and $0.022 M_{\odot}$, and the disk to star mass ratio is only $\approx 0.03$, explaining the lack of fragmentation in the circumstellar disk. The luminosity outburst in model 6 is caused by one of the fragments (shown by the yellow arrow) forming in the circumfragment disk and falling onto the star owing to a complex interplay and exchange of angular momentum with other fragments and spiral filaments.

Finally, we note that late bursts in our model are also possible if a fragment is ejected from the disk into the intrucluster medium through the multi-body gravitational interaction (Basu \& Vorobyov 2012). The ejection is paired with another fragment losing its angular momentum and falling onto the star, producing a strong accretion burst. As table 1 in Basu \& Vorobyov (2012) demonstrates, some ejection events may occur 0.7-0.8 Myr after the formation of the protostar, which is usually an optically visible phase. To summarize, most burst events in our model take place in the partly embedded Class I phase, with a smaller fraction occurring in the deeply embedded phase and a few bursts in the

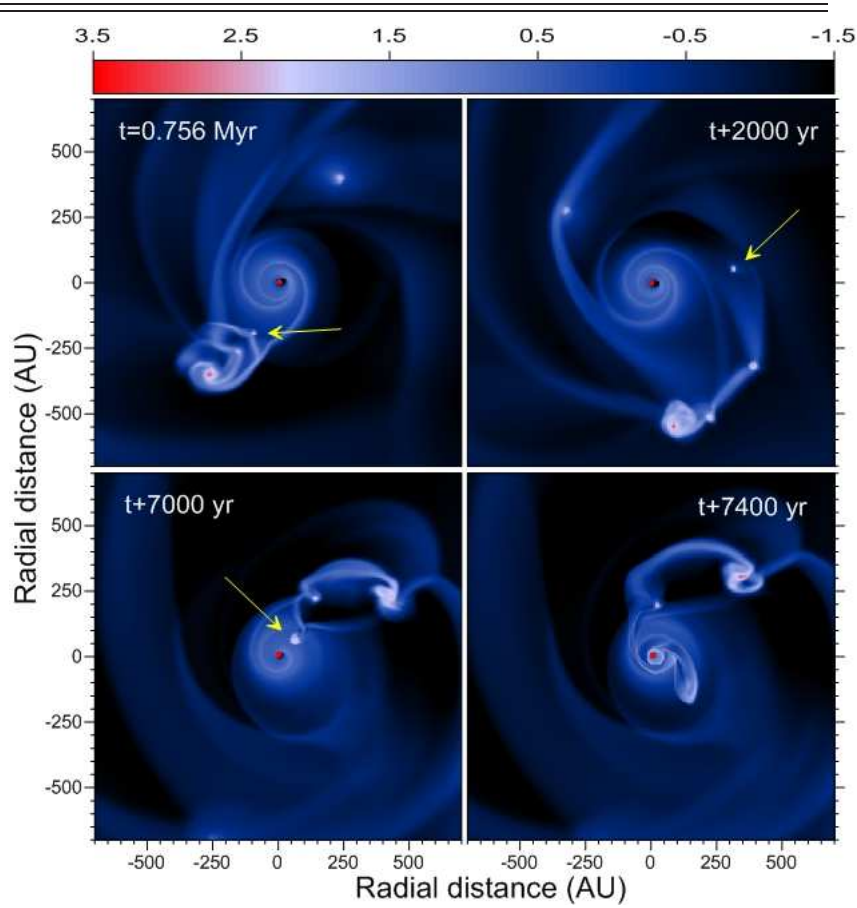

FIG. 10. - Gas surface density images (in log $\mathrm{g} \mathrm{cm}^{-2}$ ) showing the evolution in model 6 before and after the accretion burst at $t=0.763$ Myr. The yellow arrows track the position of a fragment forming in the disk of the companion and migrating into the central star. The time is counted since the beginning of gravitational collapse. The central star is formed at 0.146 Myr.

optically visible Class II phase.

The tendency of luminosity bursts to mainly occur in the embedded phase can be understood by analyzing the updates applied to our numerical hydrodynamics code. The use of Semenov opacities (instead of Bell \& Lin's) and stiffer equation of state both contribute to make disk fragmentation more difficult owing to an increased disk temperature. However, the envelope infall onto the disk in the embedded phase increases the surface density and brings periodically the disk to the fragmentation boundary. Once the envelope begins to dissipate and the infall rate drops, the burst activity subsides, explaining the scarcity of bursts in the Class II phase. The difference with our previous work here is that it now takes pre-stellar cores with somewhat higher mass and angular momentum to trigger the burst phenomenon after form- 

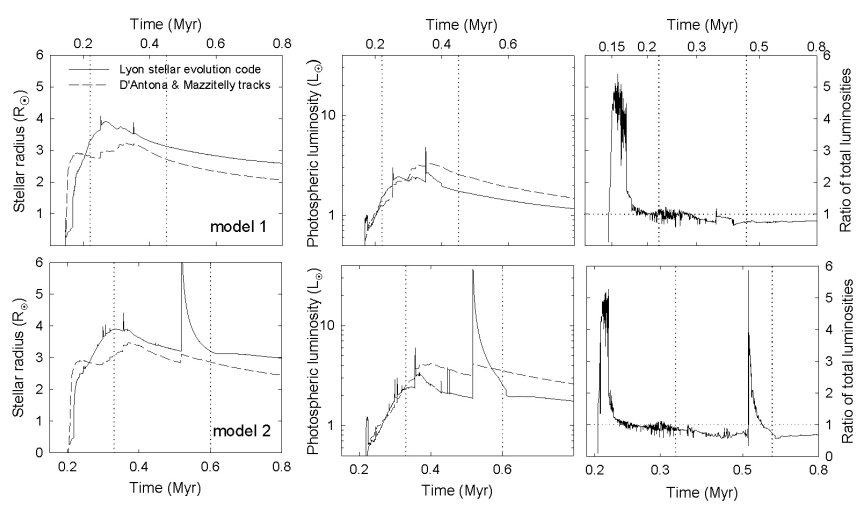

FIG. 11.- Time evolution of the stellar radius (left column), photospheric luminosity (middle column) and ratio of total luminosities $L_{*, \text { Lyon }} / L_{* \text {,DAM }}$ (right column) derived from the Lyon stellar evolution code (Baraffe \& Chabrier 2010) and the non-accreting stellar evolution tracks of D'Antona \& Mazzitelli (1994). Shown are results for model 1 (top row) and model 2 (bottom row). The vertical dotted lines mark the Class $0 / \mathrm{I}$ and Class I/II boundaries.

ing a star-disk system.

The use of the Lyon stellar evolution code (instead of precalculated tracks of D'Antona \& Mazzitelli (1994)) has a more complicated effect. The left and middle columns in Figure 11 present the time evolution of the stellar radius $R_{*}$ and photospheric luminosity $L_{*, \mathrm{ph}}$ in model 1 (top row) and model 2 (bottom row) derived using the Lyon stellar evolution code (solid lines) and D'Antona \& Mazzitelli stellar evolution tracks (dashed lines). The right column shows the ratio of total luminosities $L_{* \text {,Lyon }} / L_{* \text {,DAM }}$ found using the Lyon code and D'Antona \& Mazzitelli tracks (hereafter, DAM tracks). The vertical dotted lines mark the Class 0/I and Class I/II boundaries.

Evidently, the time evolution of $R_{*}$ is different in the Lyon code and DAM tracks. In the early Class 0 phase, the stellar radius in the Lyon code is significantly smaller than that of the DAM tracks. As a result, the accretion luminosity in the Lyon tracks is considerably greater ${ }^{11}$. At the same time, the photospheric luminosity in the Lyon code and DAM tracks are similar. The net result is that the total luminosity in the Lyon code is notably greater in the early Class 0 phase, as the right-hand-side column in Figure 11demonstrates, making disk fragmentation more difficult and reducing the burst activity in the early Class 0 phase.

In the late Class 0 phase, the stellar radius in the Lyon code increases owing to absorption of a fraction of the accretion energy and the situation reversers: $R_{*}$ in the Lyon code becomes larger than that in the DAM tracks. Steep episodic rises in $R_{*}$ seen in model 1 and especially in model 2 at $t \approx 0.55 \mathrm{Myr}$ are caused by accretion bursts, leading to stellar bloating due to the absorbed accretion energy. In the Class I phase, $R_{*}$ in the Lyon code is systematically larger than in the DAM tracks and the photospheric and accretion luminosities are smaller (apart from time instances with strong bursts). The net result is that the total luminosity in the Lyon code becomes smaller on average that that in the DAM tracks,

11 Note that when deriving the stellar radii and photospheric luminosities from the DAM tracks we use the accretion histories obtained by hydrodynamical simulations coupled with the Lyon stellar evolution code. That is why the accretion rate and stellar mass are identical in both the DAM and Lyon cases.

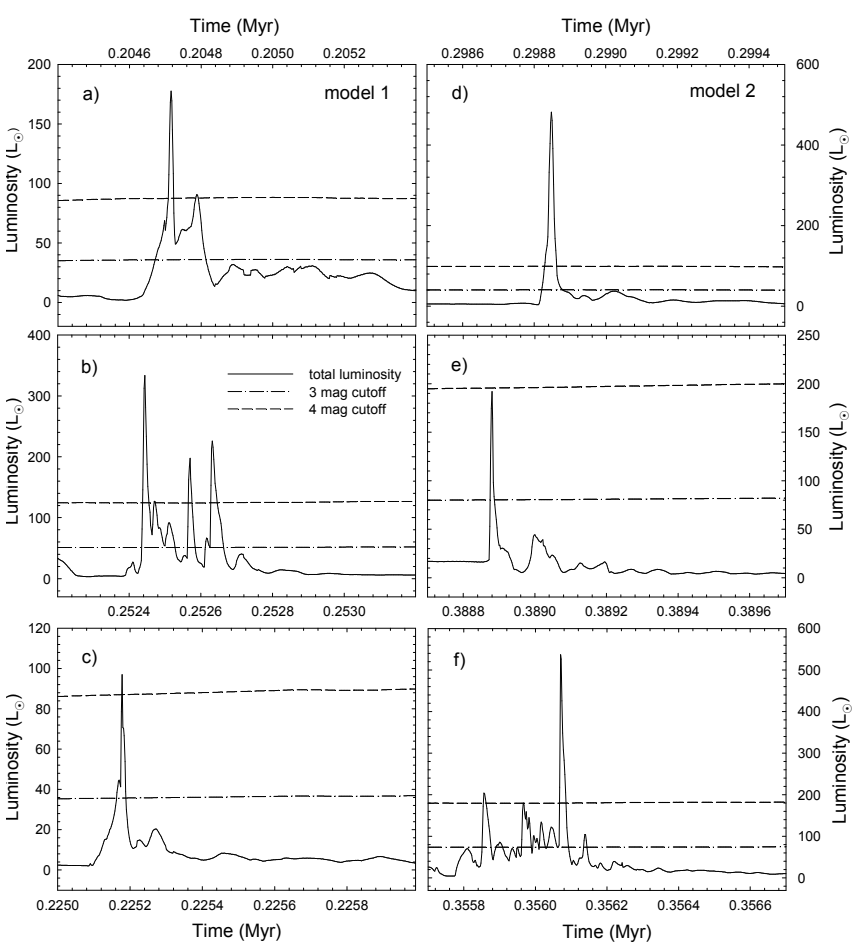

FIG. 12.- Zooming in onto individual bursts in model 1 (left column) and model 2 (right column). The solid lines are the total luminosity vs. time, while the dashed and dash-dotted lines are the 4-magnitude and 3-magnitude cutoffs.

making disk fragmentation easier. This effect, along with continuing infall from the envelope, explain why luminosity bursts tend to occur in the Class I phase.

\section{ISOLATED AND CLUSTERED LUMINOSITY BURSTS}

In this section, we zoom in onto individual luminosity bursts in our models in order to describe their evolution on short time scales. Figure 12 presents the total luminosity of individual bursts in model 1 (left column) and model 2 (right column) during six time periods, each of $10^{3} \mathrm{yr}$ in duration. Also shown with the dashed and dash-dotted lines are the 3-magnitude and 4-magnitude cutoffs to help identify the bursts (see Section 4). Two different types of bursts are evident in the figure. The first type can be described as single, isolated bursts as shown in panels $\mathbf{c}, \mathbf{d}$, and $\mathbf{e}$. These bursts are caused by compact infalling fragments, which have withstood the disruptive effect of tidal torques when approaching the central star and have passed though the sink cell almost intact. These events are usually characterized by rather short rise and fall times due to the compact nature of the fragments triggering the bursts.

The other type are closely-packed bursts, which occur one after another as shown in panels $\mathbf{a}, \mathbf{b}$, and $\mathbf{f}$. As a rule, there is one primary, most energetic burst and a few secondary bursts of lesser amplitude. These clustered bursts are caused by infalling fragments that have started to disintegrate on their approach to the star due to the disruptive influence of tidal torques. An example of such a phenomenon is illustrated in Figure 13 showing the gas surface density in model 1 in the inner region during a short time period corresponding to Figure $12 \mathbf{a}$. An extended fragment approaching the star is evident in the upper panels of the figure. At a distance of a few tens AU from the star, the fragment loses its roundish 


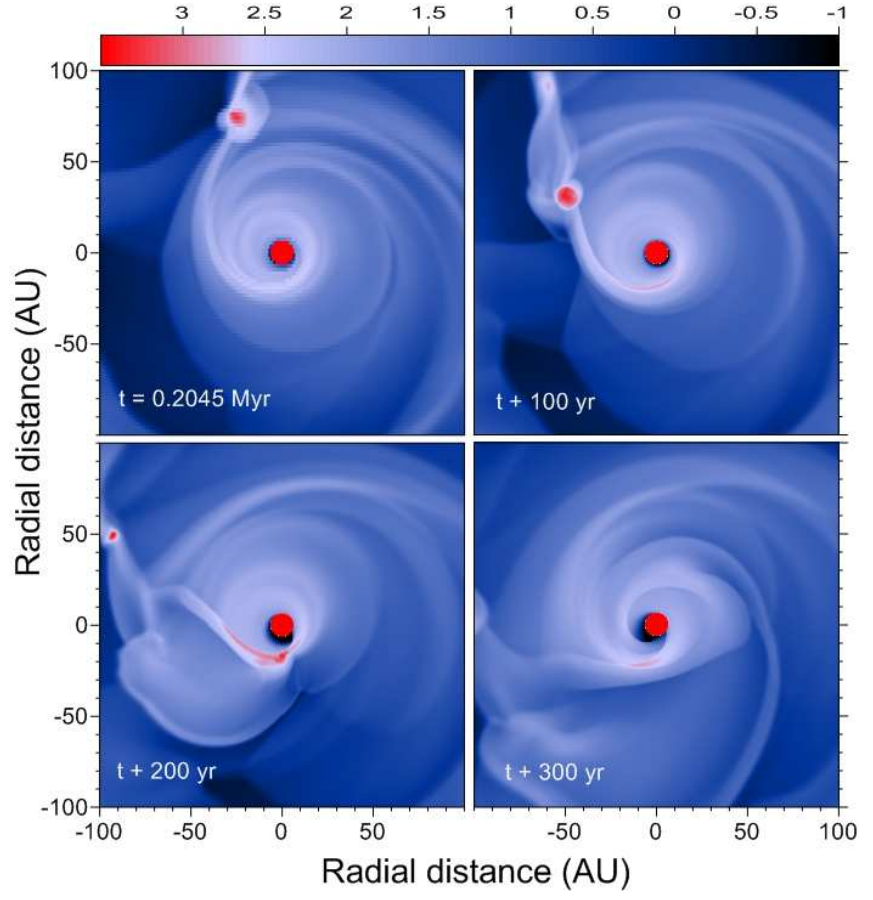

FIG. 13. - Zoom-in onto a fragment approaching the central star. Shown is the gas surface density (in log $\mathrm{g} \mathrm{cm}^{-2}$ ) during a short time period corresponding to a clustered burst in Figure $12 \mathbf{a}$. The star is marked by the red circle in the coordinate center.

shape, stretches into a clumpy filament, and finally accretes through the sink sell onto the star. It is interesting to note that another fragment seen in the bottom-left panel is hurled to a larger distance due to the gravitational exchange of angular momentum with the infalling fragment, a phenomenon that can potentially contribute to the survival probability of fragments in the disk. Depending on the final structure of the filament, two or more bursts of varying amplitude can be triggered.

The typical times between individual bursts in the clustered-burst event are 30-100 yr, too long to be firmly detected on the basis of available FUori observations. Nevertheless, the knotty structure seen in many protostellar jets and spacing between the knots are consistent with short periods of variability in the mass accretion rates as could be expected from clustered bursts.

Another class of young pre-main-sequence stars, EXors (known after its prototype star EX Lupi), are known for exhibiting repetitive bursts of lesser magnitude on timescales of several years (Hartmann \& Kenyon 1996; Audard et al. 2014). Until recently, the repetitive nature of EXor bursts has been considered as a defining characteristic of such objects. The existence of clustered bursts, if confirmed, could further erode the ever shrinking gap between FUors and EXors. In this context, post-burst observations of known FUors using (sub)-millimeter interferometers may search for a clumpy filamentary structure in the inner 20-30 AU caused by a disintegrated fragment approaching the star. A successful detection of such a structure would imply another outburst to come.

\section{THE FRACTION OF BURST-PRODUCING CORES}

Finally, based on our numerical simulations, we want to estimate the fraction of star-forming cores that is expected to produce bursts after forming a star-disk system. Our model with core mass $M_{\text {core }}=0.3 M_{\odot}$ does not undergo a burst mode driven by the formation of large fragments in the disk, however the models we run with $M_{\text {core }}=1.1 M_{\odot}$ and greater mass do show significant bursts with mass accretion rate exceeding $10^{-4} M_{\odot} \mathrm{yr}^{-1}$. Figure 6 of Basu \& Vorobyov (2012) shows a correlation between fragmentation events, core mass, and $\beta$. Despite limitations of exploration of parameter space in these models, we can combine the information there with the observational finding of Caselli et al. (2002) that $\beta$ has a median value of 0.02 and most values in the range $\sim 10^{-4}-10^{-1}$ to conclude that most cores with $M_{\text {core }} \geq 1.0 M_{\odot}$ will exhibit fragmentation and bursts and that most cores with $M_{\text {core }} \leq 0.5 M_{\odot}$ will not exhibit bursts.

To make an estimate of what number fraction of starforming events these represent, we can further employ the stellar initial mass function (IMF) of Chabrier (2005) and the idea that the core mass function is simply the same shape as the IMF but scaled up by a multiplicative factor of about 3 (see, e.g., André et al. 2009). In this case, and using $0.075 M_{\odot}$ as a minimum stellar mass (Chabrier \& Baraffe 2000), we can identify the number of cores with $M_{\text {core }} \geq 1.0 M_{\odot}$ with the number of stars with mass $m \geq 0.33 M_{\odot}$. Their number fraction can be found easily using the cumulative function of stars introduced by Basu et al. (2014) in their equation (15):

$$
\begin{aligned}
& F(m)=\frac{1}{2} \operatorname{erfc}\left(-\frac{\ln (m)-\mu_{0}}{\sqrt{2} \sigma_{0}}\right) \\
& -\frac{1}{2} \exp \left(\omega \mu_{0}+\frac{\omega^{2} \sigma_{0}^{2}}{2}\right) m^{-\omega} \operatorname{erfc}\left(\frac{\omega \sigma_{0}}{\sqrt{2}}-\frac{\ln (m)-\mu_{0}}{\sqrt{2} \sigma_{0}}(1) 4\right)
\end{aligned}
$$

where $\operatorname{erfc}(x)=1-\operatorname{erf}(x), \operatorname{erf}(x)$ is the Error function, $\omega$ is the power-law index of the high mass tail of the mass distribution function $\left(\propto m^{-(1+\omega)}\right)$, and $\mu_{0}$ and $\sigma_{0}$ are additional parameters that help define the mean and variance of the distribution.

Their best fit to the Chabrier IMF shows that about $40 \%$ of all stellar objects have $m \geq 0.33 M_{\odot}$, hence presumed to originate from a core with mass $M_{\text {core }} \geq$ $1.0 M_{\odot}$. The criterion $M_{\text {core }} \leq 0.5 M_{\odot}$ for no bursts will translate to $0.075 M_{\odot} \leq m \leq 0.167 M_{\odot}$ in the stellar IMF, and this accounts for some $32 \%$ of all stellar objects. If $40 \%$ of stellar objects are expected to definitely have bursts and $32 \%$ are not, this leaves an intermediate $28 \%$ that may have bursts depending on the level of rotation in the initial core. Therefore, we can say that the fraction of star-forming cores that can be expected to display bursts after forming a star-disk system falls somewhere in the range of $40 \%-70 \%$.

\section{CONCLUSIONS}

We have shown that variable protostellar accretion with episodic bursts is a robust property of protostellar collapse. This phenomenon is often present in a collapse environment in which a protostellar disk has a self-consistent mass loading from the core envelope. An accurate calculation of photospheric and accretion luminosities, as well as improved disk thermal physics and dust opacities, have refined our numerical hydrodynamics model, enabling better characterization of luminosity bursts caused by disk gravitational fragmentation followed by fragments migrating onto the protostar. 
In agreement with our previous studies, we found that an increase in the initial core mass and angular momentum favours the burst phenomenon, while higher levels of background radiation and magnetic fields can moderate the burst activity. A minimum mass infall rate onto the disk on the order of a few $\times 10^{-6} M_{\odot} \mathrm{yr}^{-1}$ is required to generate the bursts. The main results can be summarized as follows.

- A general correlation between the amplitude of time variations in $\dot{M}$ and the strength of nonaxisymmetric perturbations in the disk, as defined by the global Fourier amplitudes, suggests a causal link between accretion variability and the disk gravitational instability. In our models, long-term variations in protostellar accretion are caused by the nonlinear interaction between different spiral modes in the gravitationally unstable disk, while episodic accretion bursts are triggered by fragments migrating onto the star.

- Most luminosity bursts in our models occur in the partly embedded Class I phase, with a smaller fraction taking place in the deeply embedded Class 0 phase and a few occasional bursts in the optically visible Class II phase.

- The properties of the bursts are found to be in good agreement with those inferred for FU-Orionis-type objects (FUors). For instance, our models yield the mean luminosity and average burst duration of $312 L_{\odot}$ and $39 \mathrm{yr}$, respectively, only a factor of 1.5-2 higher than in FUors.

- Depending on the ability of fragments to withstand the tidal torques when approaching the star, two types of bursts can occur: the isolated and clustered ones. In the former, the fragment is accreted almost intact, producing a well-defined burst, while in the latter the fragment is stretched into a knotty filament when approaching the star, thus producing a series of closely-packed bursts of varying amplitude separated from each other by several decades.

- Adopting the stellar IMF of Chabrier (2005) and assuming that the core mass function is the same shape as the IMF but is scaled up by a factor of about 3, we estimate that about $40 \%-70 \%$ of the star-forming cores can be expected to exhibit bursts after forming a star-disk system.

In the present work, the mass accretion rates $\dot{M}$ were calculated at the position of the inner sink cell at $6 \mathrm{AU}$. We have shown that the time behavior of $\dot{M}$ is similar at $8 \mathrm{AU}$ and $12 \mathrm{AU}$, thus demonstrating that the accretion variability and the bursts are not the artifacts of the adopted inner boundary condition in our numerical simulations. Nevertheless, more work is needed to develop a self-consistent link between the inner and outer disk regions.

\section{ACKNOWLEDGMENTS}

The authors are thankful to the anonymous referee for providing critical comments that helped to improve the manuscript. The authors are thankful to Isabelle Baraffe and Gilles Chabrier for providing the stellar evolution code. This work is partly supported by the RFBR grant 14-02-00719. The simulations were performed on the Shared Hierarchical Academic Research Computing Network (SHARCNET), on the Atlantic Computational Excellence Network (ACEnet), and on the Vienna Scientific Cluster (VSC-2). SB was supported by a Discovery Grant from the Natural Sciences and Engineering Research Council (NSERC) of Canada.

\section{REFERENCES}

Ábrahám, P., Kóspál, A., Csizmadia, S., et al. 2004, A\&A, 419, L39

André, P., Ward-Thompson, D., \& Barsony, M. 1993, ApJ, 406, 122

André, P., Basu, S., \& Inutsuka, S.-i. 2009, in Structure Formation in Astrophysics, ed. G. Chabrier, (Cambridge Univ. Press, Cambridge), 254

Arce, H. G., Mardones, D., Corder, S. A., Garay, G., NoriegaCrespo, A., \& Raga, A. C. 2013, ApJ, 774, 39

Armitage, P.J., Livio, M., \& Pringle, J.E. 2001, MNRAS, 324, 705

Audard, M., et al. 2014, in Protostars and Planets VI, eds. H. Beuther, R. S. Klessen, C. P. Dullemond, \& T. Henning, University of Arizona Press, 387

Baraffe, I., Chabrier, G., \& Gallardo, J. 2009, ApJ, 702, L27

Baraffe, I., \& Chabrier, G. 2010, A\&A, 521, 44

Baraffe, I., Vorobyov, E. I., \& Chabrier, G. 2012, ApJ, 756, 118

Basu, S., Gil, M., \& Auddy, S. 2014, MNRAS, accepted (arXiv:1503.00023)

Basu, S., \& Vorobyov, E. I. 2012, ApJ, 750, 30

Bate, M., Lodato, G., Pringle, J. E., 2014, MNRAS, 401, 1505

Basu, S. 1997, ApJ, 485, 240

Bell, K. R., Lin, D. N. C., 1994, ApJ, 427, 987

Billot, N., Morales-Calderón, M., Stauffer, J. R., Megeath, S. T., \& Whitney, B. 2012, ApJL, 753, 35

Boley, A. C., Hayfield, T., Mayer, L., \& Durisen, R. H. 2010, Icarus, 207, 509

Caselli, P., Benson, P. J., Myers, P. C., \& Tafalla, M. 2002, ApJ, 572,238

Cha, S.-H. \& Nayaksin, S. 2011, MNRAS, 415, 3319
Chen, H., Myers, P. C., Ladd, E. F., \& Wood, D. O. S. 1995, ApJ, 445,377

Chabrier, G. 2005, in The Initial Mass Function 50 years later, ed. E. Corbelli, F. Palla, and H. Zinnecker, (Springer, Dordrecht), 41

Chabrier, G. \& Baraffe, I. 2000, ARA\&A, 38, 337

D’Antona, F., \& Mazzitelli, I. 1994, ApJS, 90, 467

Dapp, W. B., \& Basu, S. 2009, MNRAS, 395, 1092

Dunham M. M., Evans N. J., II, Terebey S., Dullemond C. P., Young C. H., 2010, ApJ, 710, 470

Dunham, M. M., \& Vorobyov, E. I. 2012, ApJ, 747, 52

Dunham, M. M., et al. 2014, in Protostars and Planets VI, eds. H. Beuther, R. S. Klessen, C. P. Dullemond, \& T. Henning, University of Arizona Press, 195

Evans, N. J., II, Dunham, M. M., Jorgensen, J. K., et al. 2009, ApJS, 181, 321

Foster, P. N., Chevalier, R. A. 1993, ApJ, 416, 303

Hartmann, L., \& Kenyon, S. J. 1996, ARAA, 34, 207

Johnson, B. M., \& Gammie C. F. 2003, ApJ, 597, 131

Kenyon, S. J., Hartmann, L. W., Strom, K. M., \& Strom, S. E. 1990, ApJ, 99, 869

Kim, H. J., Evans, N. J., II, Dunham, M. M., Lee, J.-E., \& Pontoppidan, K. M. 2012, ApJ, 758, 38

Kośpál, A., Ábrahám, P., Acosta-Pulido, J. A., et al. 2011, A\&A, 527,133

Larson, R. B. 1969, MNRAS, 149, 271

Lee, J.-E. 2007, J. Korean Astron. Soc., 40, 83

Machida, M. N., Inutsuka, S., \& Matsumoto, T. 2011, ApJ, 729, 42

Masunaga, H., \& Inutsuka, S.-I. 2000, ApJ, 531, 350 
Meru, F., \& Bate, M. R. 2012, MNRAS, 427, 2012

Nakano, T., \& Nakamura, T. 1978, PASJ, 30, 671

Nayakshin, S., \& Lodato, G. 2012, MNRAS, 426, 70

Ohtani, T., Kimura, S. S., Tsuribe, T., Vorobyov, E. I. 2014, PASJ, in press

Padoan, P., Haugbolle, T., Nordlung A. 2014, arXiv:1407.1452

Penston, M. V. 1969, MNRAS, 144, 425

Robitaille, T. P., Whitney, B. A., Indebetouw, R., Wood, K., \& Denzmore, P. 2006, ApJS, 167, 256

Quanz S. P., et al. 2007, ApJ, 668, 359.

Rice, W. K. M., Mayo, J. H., \& Armitage, P. J. 2010, MNRAS, 402, 1740

Scholz, A., Froebrich, D., \& Wood, K. 2013, MNRAS, 430, 2910

Michael, S. \& Durisen, R. H. 2010, MNRAS, 406, 273

Millan-Gabet R., et al. 2006, ApJ, 641, 547

Semenov, D., Henning, Th., Helling, Ch., Ilgner, M., \& Sedlmayr, E. 2003, A\&A, 410, 611 .

Scholz A., Froebrich, D., Wood, K. 2013, MNRAS, 430, 2910

Shu, F. S. 1977, ApJ, 214, 488

Stamatellos, D., Whitworth, A. P., \& Hubber, D. A. 2011, ApJ, 730,32
Tsukamoto, Y., Takahashi, S. Z., Machida, M. N., Inutsuka, S. 2014, ArXiv:1404:7271

Visser, R., \& Bergin, E. A. 2012, ApJ, 754, 18

Vorobyov, E. I. 2009, ApJ, 704, 715

Vorobyov, E. I. 2010, ApJ, 723, 1294

Vorobyov, E. I. 2011, ApJ, 729, 146

Vorobyov, E. I. 2013, A\&A, 552, 129

Vorobyov, E. I., \& Basu, S. 2005, MNRAS, 360, 675

Vorobyov, E. I., Basu, S., 2006, ApJ, 650, 956

Vorobyov, E. I., \& Basu, S. 2009, MNRAS, 393, 822

Vorobyov, E. I., \& Basu, S. 2010a, ApJ, 719, 1896

Vorobyov, E. I., \& Basu, S. 2010b, ApJL, 714, 133

Vorobyov, E. I., Baraffe, I., Harries, T., Chabrier, G. 2013, A\&A, 557,35

Vorobyov, E. I., Zakhozhay, O. V., Dunham, M. M. 2013, MNRAS, 433,3256

Zhu, Z., Hartmann, L., \& Gammie, C. 2009, ApJ, 694, 1045

Zhu, Z., Hartmann, L., Nelson, R. P., \& Gammie, C. F. 2012, ApJ, 746,110 